\title{
Sensitivity Of The Stochastic Response Of Structures Coupled With Vibrating Barriers
}

\author{
A.TOMBARI ${ }^{1}$, I. ZENTNER ${ }^{1}$ and P. CACCIOLA ${ }^{2}$ \\ ${ }^{1}$ Institut des Sciences de la Mécanique et Applications Industrielles / UMR 9219 CNRS-EDF-CEA-ENSTA, 1, Avenue \\ du Général de Gaulle92141 CLAMART Cedex, France. \\ ${ }^{2}$ School of Environment and Technology, University of Brighton, Cockcroft Building Lewes Road BN2 4GJ, Brighton, \\ $U K$.
}

The sensitivity of the stochastic response of linear behaving structures controlled by the novel Vibrating Barrier (ViBa) device is scrutinized. The Vibrating Barrier (ViBa) is a massive structure, hosted in the soil, calibrated for protecting structures by exploiting the structure-soilstructure interaction effect. Therefore the paper addresses the study of the sensitivity of soilstructure coupled systems in which the soil is modelled as a linear elastic medium with hysteretic damping. In order to accomplish efficient sensitivity analyses, a reduced model is determined by means of the Craig-Bampton procedure. Moreover, a lumped parameter model is used for converting the hysteretic damping soil model rigorously valid in the frequency domain to the approximately equivalent viscous damping model in order to perform conventional time-history analysis. The sensitivity is evaluated by determining a semi-analytical method based on the dynamic modification approach for the case of multi-variate stochastic input process. The ground motion is modelled as non-stationary zero-mean Gaussian random process defined by a given evolutionary Power Spectral Density function. The paper presents the sensitivity of the response statistics of a model of an industrial building, passively controlled by the ViBa, to relevant design parameters. Comparisons with pertinent Monte Carlo Simulation will show the effectiveness of the proposed approach.

Keywords: Structure-Soil-Structure Interaction, hysteretic damping, Craig-Bampton method, sensitivity analysis, stochastic response, Vibrating Barriers. 


\section{Introduction}

Unpredicted vibrations due to ground motion earthquakes cause severe damages to the structural components that lead to the deterioration or collapse of buildings. Although several techniques and strategies of Vibration Control can be adopted for the seismic design of new structures or seismic retrofit of existing buildings, every approach is based on the direct design or intervention on the members or on the control systems belonging to the structure. Conversely, for heritage buildings, strong interventions are avoidable to preserve the authenticity and integrity of the historic character of the monument; moreover, most of the existing private buildings are seismically deficient requiring an important cost impact for their seismic protection. In this context, a novel passive control device called Vibrating Barrier (ViBa), has been recently proposed by Cacciola (2012). The Vibrating Barrier is a massive structure, hosted in the soil and detached from the other structures, calibrated for absorbing portion of the ground motion input energy. The aim is to reduce the vibrations of neighborhood structures by exploiting the structure-soil-structure interaction (SSSI) effect, i.e. the dynamic influence among vibrating structures caused by the wave propagation through the soil. To achieve this goal, the proper calibration of the ViBa parameters is required. Readers can refers to the work of Cacciola et al. (2015) and Cacciola and Tombari (2015) for an in-depth study of the ViBa. In this regard, uncertainty clearly plays a relevant role in the ViBa design. The uncertainties such as the random nature of the seismic action and the dispersion of the mechanical properties of both the structure and the soil result in a substantial difference between the actual and the computed seismic response. Therefore, sensitivity analysis is crucial to understand the impact of inaccurate model parameters on the structural response.

Probably one the earliest contribution to sensitivity analysis in structural mechanics are due to Fox and Kapoor (1968). In the framework of stochastic mechanics several papers have been devoted to study the sensitivity of the response of structural systems subjected to stochastic excitations (see e.g. 
Szopa 1985, Hien and Kleiber 1991, Huang and Socha 1993, Huang and Soong 1994, Benfratello et al. 2000). More recently, Bhattacharyya and Chakraborty (2002) extended Neumann expansion method within the framework of Monte Carlo simulation for sensitivity analysis of dynamic systems subjected to ground motion acceleration modelled by a stationary process. Chaudhuri and Chakraborty (2004) determined the response sensitivity in the frequency domain of structures subjected to nonstationary seismic processes. Cacciola et al. (2005) proposed a method to determine the sensitivity of second order statistics of linear structures forced by Gaussian excitation. Marano et al. (2008) performed a parametric sensitivity analysis of the spectral response of a stochastic SDOF system subjected to a nonstationary seismic action with respect to uncertain soil parameters. Liu (2012a,b) and Liu and Paavola (2014) proposed numerical methods for calculation of the sensitivity and Hessian matrix of the response of structures subjected to uniformly modulated evolutionary random excitation. Johnson and Wojtkiewicz (2014), proposed a high computationally efficient approach for the computation of the sensitivities of power spectral densities, and mean-square responses of a structural system. Recently, Sarkar and Ghosh (2015) proposed a hybrid method to study the sensitivity of the stochastic response of linear behaving structures considering both uncertainties in the structural parameters and in the external input.

In this paper, the semi-analytical modal procedure proposed by Cacciola et al. (2005), has been extended in order to consider multi-variate Gaussian stochastic load process. The method allows the evaluation of the sensitivity of the nodal response of large MDOF systems in the modal space corresponding to the nominal values. In this regard, the method proposed in this paper allows the sensitivity analysis in the reduced model derived by means of the Craig-Bampton procedure (Bampton and Craig 1968). The effects of interaction between the structure and the soil, namely the soil-structure interaction (SSI), are considered according to the substructure approach proposed by Kausel (1978) in which the soil is simulated by dynamic impedances subjected to seismic forces. The soil impedances are contained in the dynamic stiffness matrix computed by boundary element method (BEM) and 
accounts for hysteretic soil damping (Neumark 1957). Furthermore, a Lumped Parameter Model (LPM) composed of frequency-independent parameters, is used for converting the exact soilfoundation reference hysteretic model formulated in the frequency domain to an approximately equivalent viscous model in the time domain. Time domain sensitivity analysis of the stochastic response through the LPM reduced model. Finally, numerical studies are carried out for investigating the stochastic response of a model of an Industrial Building passively controlled by the novel Vibrating Barrier device with respect to relevant design parameters.

\section{Problem formulation of the global model}

Consider the large global n-degree of freedom (n-DOF) structural linear system depicted in Figure 1. The dynamic governing equations of motion are casted in the frequency domain as follows:

$$
\left\{\mathbf{K}_{\text {glob }}(\omega)-\omega^{2} \mathbf{M}_{\text {glob }}+i \omega \mathbf{C}_{\text {glob }}\right\} \mathbf{u}(\omega)=\mathbf{f}(\omega)
$$

where $i=\sqrt{-1} ; \mathbf{M}_{\text {glob }}, \mathbf{C}_{\text {glob }}$, and $\mathbf{K}_{\text {glob }}(\omega)$ are the real [n $\mathrm{x} \mathrm{n}$ ] global mass, damping and stiffness matrices respectively; $\mathbf{u}(\omega)$ and $\mathbf{f}(\omega)$ corresponds to the [n $\mathrm{x} 1$ ] vectors of the nodal absolute displacements and the applied forces in the frequency domain ( $\omega$ is the circular frequency).

The global system is partitioned in three subdomains or sub-structures, namely the structure to be protected hereafter referred in the paper by the subscript $[\cdot]_{\text {str }}$, the ViBa device, indicated by the subscript $[\cdot]_{\mathrm{ViBa}}$, and the soil-foundations interface denoted by $[\cdot]_{\mathrm{SF}}$.

Therefore, Eq. (1) is restated as:

$$
\begin{aligned}
\left\{\left[\begin{array}{ccc}
\mathbf{K}_{\mathrm{ViBa}} & \mathbf{0} & \mathbf{K}_{\mathrm{ViBa}, \mathrm{SF}} \\
\mathbf{0} & \mathbf{K}_{\mathrm{str}} & \mathbf{K}_{\mathrm{str}, \mathrm{SF}} \\
\mathbf{K}_{\mathrm{SF}, \mathrm{ViBa}} & \mathbf{K}_{\mathrm{SF}, \mathrm{str}} & \mathbf{K}_{\mathrm{SF}}
\end{array}\right]-\omega^{2}\left[\begin{array}{ccc}
\mathbf{M}_{\mathrm{ViBa}} & \mathbf{0} & \mathbf{M}_{\mathrm{ViBa}, \mathrm{SF}} \\
\mathbf{0} & \mathbf{M}_{\mathrm{str}} & \mathbf{M}_{\mathrm{str}, \mathrm{SF}} \\
\mathbf{M}_{\mathrm{SF}, \mathrm{ViBa}} & \mathbf{M}_{\mathrm{SF}, \mathrm{str}} & \mathbf{M}_{\mathrm{SF}}
\end{array}\right]\right. \\
\left.+i \omega\left[\begin{array}{ccc}
\mathbf{C}_{\mathrm{ViBa}} & \mathbf{0} & \mathbf{C}_{\mathrm{ViBa}, \mathrm{SF}} \\
\mathbf{0} & \mathbf{C}_{\mathrm{str}} & \mathbf{C}_{\mathrm{str}, \mathrm{SF}} \\
\mathbf{C}_{\mathrm{SF}, \mathrm{ViBa}} & \mathbf{C}_{\mathrm{SF}, \mathrm{str}} & \mathbf{C}_{\mathrm{SF}}
\end{array}\right]\right\}\left[\begin{array}{c}
\mathbf{u}_{\mathrm{ViBa}}(\omega) \\
\mathbf{u}_{\mathrm{str}}(\omega) \\
\mathbf{u}_{\mathrm{SF}}(\omega)
\end{array}\right]=\left[\begin{array}{c}
\mathbf{0} \\
\mathbf{0} \\
\mathbf{f}_{\mathrm{SF}}(\omega)
\end{array}\right]
\end{aligned}
$$


The vector $\mathbf{u}(\omega)$ is hence divided into the [p x 1]-vector of the $\mathrm{ViBa}, \mathbf{u}_{\mathrm{ViBa}}$, the [q $\mathrm{x} 1$ 1]-vector of the structure, $\mathbf{u}_{\text {str }}$, and the [ $\mathrm{r} \times 1$ ]-vector of the soil-foundations system $\mathbf{u}_{\mathrm{SF}}$. The mass $\mathbf{M}_{\mathrm{str}}$, damping $\mathbf{C}_{\text {str }}$ and stiffness $\mathbf{K}_{\text {str }}$ [q $\mathrm{x}$ q]-matrices of the structure are derived by the traditional finite element approach as well the [p x p]-matrices, $\mathbf{M}_{\mathrm{ViBa}}, \mathbf{C}_{\mathrm{ViBa}}, \mathbf{K}_{\mathrm{ViBa}}$ of the ViBa. The [r x r]-matrices $\mathbf{M}_{\mathrm{SF}}$, $\mathbf{C}_{\mathrm{SF}}$, and $\mathbf{K}_{\mathrm{SF}}$ are the matrices of the nodes at the soil-foundations interface determined by the substructure approach proposed by Kausel (1978); by defining $\mathbf{K}_{\mathrm{dyn}}(\omega)$ as the dynamic stiffness matrix, that can be decomposed in the real part (Re) and imaginary part (Im) as:

$$
\mathbf{K}_{\mathrm{dyn}}(\omega)=\operatorname{Re}\left\{\mathbf{K}_{\mathrm{dyn}}(\omega)\right\}+i \operatorname{Im}\left\{\mathbf{K}_{\mathrm{dyn}}(\omega)\right\}
$$

the following relations are derived: $\mathbf{M}_{\mathrm{SF}}=\mathbf{M}_{\mathrm{F}}, \mathbf{C}_{\mathrm{SF}}=\mathbf{C}_{\mathrm{F}}+\operatorname{Im}\left\{\mathbf{K}_{\text {dyn }}(\omega)\right\} / \omega$ and $\mathbf{K}_{\mathrm{SF}}=\mathbf{K}_{\mathrm{F}}+\operatorname{Re}\left\{\mathbf{K}_{\mathrm{dyn}}(\omega)\right\} ; \mathbf{M}_{\mathrm{F}}, \mathbf{C}_{\mathrm{F}}$, and $\mathbf{K}_{\mathrm{F}}$ the mass, damping and stiffness [r x r]-matrices of the foundation itself, respectively.

The dynamic stiffness matrix $\mathbf{K}_{\text {dyn }}(\omega)$ is determined in order to take into account the effects of the soil, such as the soil-foundation interaction (SFI), the foundation-soil-foundation interaction (FSFI), the hysteretic damping as well as the radiation (or geometric) damping without resorting to a large finite element model of the soil. The dynamic impedance matrix is computed by condensing out the entire soil-foundations system onto the foundation interfaces in the frequency domain. It relates the displacements in the nodes on the structure-soil interface to the interaction forces $\mathbf{f}_{\mathrm{s}}(\omega)$ of the unbounded soil. Both dynamic impedance matrix $\mathbf{K}_{\mathrm{dyn}}(\omega)$ and the interaction force vector $\mathbf{f}_{\mathbf{s}}(\omega)$ are obtained from linear elastodynamic problems solved by means of Boundary Element Method (BEM) approach. BEM is based on the validity of the superposition principle and hence, it is conveniently formulated in the frequency domain where hysteretic damping is rigorously valid because of its noncausal nature (Crandall 1970, Spanos and Zeldin 2000).

The vector $\mathbf{f}_{\mathrm{SF}}(\omega)$ collects the loads at the soil-foundation interface due to the free-field motion as follows: 


$$
\mathbf{f}_{\mathrm{SF}}(\omega)=\mathbf{f}_{\mathrm{s}}(\omega) \mathrm{u}_{\mathrm{g}}(\omega)
$$

where $\mathbf{f}_{\mathbf{s}}(\omega)$ is the $\left[\begin{array}{lll}\mathrm{r} & 1\end{array}\right]$ seismic force vector calculated at the interface for an unit harmonic displacement by means of the BEM analysis and $u_{g}(\omega)$ is the free field motion displacement at the ground surface.

\section{Determination of the reduced model}

In this paper, the Craig-Bampton (Bampton and Craig 1968) reduction method for modal substructuring is applied in order to determine the reduced model used to perform efficient timedomain sensitivity analysis. The first step involves the conversion of both impedance matrix, $\mathbf{K}_{\text {dyn }}(\omega)$, and the interaction forces $\mathbf{f}_{\mathrm{s}}(\omega)$ from the frequency into the time domain by adopting the lumped-parameter model (LPM) approach. The LPM is constituted by a combination of frequency independent springs, dashpots and masses opportunely calibrated in order to simulate the dynamic behavior in the frequency domain consistent to that obtained from the BEM analysis. The calibration of the parameters of the LPM contained in the matrices $\mathbf{K}_{\mathrm{LPM}}, \mathbf{M}_{\mathrm{LPM}}$, and $\mathbf{C}_{\mathrm{LPM}}$, is based on the approximation of each frequency-dependent component of the impedance matrix $\mathbf{K}_{\mathrm{dyn}}(\omega)$ by means of a least-square regression procedure as follows:

$$
\mathrm{K}_{\mathrm{dyn}}^{\mathrm{j}, \mathrm{k}}(\omega) \cong \mathrm{K}_{\mathrm{LPM}}^{\mathrm{j}, \mathrm{k}}-\omega^{2} \mathrm{M}_{\mathrm{LPM}}^{\mathrm{j}, \mathrm{k}}+i \omega \mathrm{C}_{\mathrm{LPM}}^{\mathrm{j}, \mathrm{k}}(\mathrm{j}, \mathrm{k}=1, \ldots, \mathrm{r})
$$

in a given frequency range $0<\omega<\omega_{\text {cut_off. }}$ Therefore, by using the frequency-independent matrices of Eq. (5), Eq. (1) can be converted in the time domain as follows:

$$
\mathbf{M} \ddot{\mathbf{u}}(\mathrm{t})+\mathbf{C} \dot{\mathbf{u}}(\mathrm{t})+\mathbf{K u}(\mathrm{t})=\mathbf{f}(\mathrm{t})
$$

where $\ddot{\mathbf{u}}(\mathrm{t}), \dot{\mathbf{u}}(\mathrm{t})$, and $\mathbf{u}(\mathrm{t})$ corresponds to the [n $\mathrm{x} 1]$ vectors of the nodal absolute accelerations, velocities and displacements as functions of time $t$, and $\mathbf{f}(t)$ is the [n x 1] vector of nodal time-varying applied forces derived by the inverse Fourier transform of the force vector $\mathbf{f}(\omega)$ of Eq. (1). 
The mass, damping and stiffness matrix namely, $\mathbf{M}, \mathbf{C}$, and $\mathbf{K}$ are the approximated matrices of their corresponding $\mathbf{M}_{\text {glob }}, \mathbf{C}_{\text {glob }}$, and $\mathbf{K}_{\text {glob }}$ of Eq. (2) where the following relations are used:

$$
\begin{gathered}
\mathbf{M}_{\mathrm{SF}}=\mathbf{M}_{\mathrm{F}}+\mathbf{M}_{\mathrm{LPM}} \\
\mathbf{C}_{\mathrm{SF}}=\mathbf{C}_{\mathrm{F}}+\frac{\operatorname{Im}\left\{\mathbf{K}_{\mathrm{dyn}}(\omega)\right\}}{\omega} \cong \mathbf{C}_{\mathrm{F}}+\mathbf{C}_{\mathrm{LPM}} \\
\mathbf{K}_{\mathrm{SF}}=\mathbf{K}_{\mathrm{F}}+\operatorname{Re}\left\{\mathbf{K}_{\mathrm{dyn}}(\omega)\right\} \cong \mathbf{K}_{\mathrm{F}}+\mathbf{K}_{\mathrm{LPM}}
\end{gathered}
$$

$\mathbf{K}_{\mathrm{F}}, \mathbf{M}_{\mathrm{F}}$ and $\mathbf{C}_{\mathrm{F}}$ are the stiffness, the mass and the viscous damping [ $\mathrm{r} \times \mathrm{r}$ ]-matrices of the foundation itself. Eq. (6) can be rewritten in the following form:

$$
\begin{aligned}
{\left[\begin{array}{ccc}
\mathbf{M}_{\mathrm{ViBa}} & \mathbf{0} & \mathbf{M}_{\mathrm{ViBa}, \mathrm{SF}} \\
\mathbf{0} & \mathbf{M}_{\mathrm{str}} & \mathbf{M}_{\mathrm{str}, \mathrm{SF}} \\
\mathbf{M}_{\mathrm{SF}, \mathrm{ViBa}} & \mathbf{M}_{\mathrm{SF}, \mathrm{str}} & \mathbf{M}_{\mathrm{F}}+\mathbf{M}_{\mathrm{LPM}}
\end{array}\right] \ddot{\mathbf{u}}(\mathrm{t})+\left[\begin{array}{ccc}
\mathbf{C}_{\mathrm{ViBa}} & \mathbf{0} & \mathbf{C}_{\mathrm{ViBa}, \mathrm{SF}} \\
\mathbf{0} & \mathbf{C}_{\mathrm{str}} & \mathbf{C}_{\mathrm{str}, \mathrm{SF}} \\
\mathbf{C}_{\mathrm{SF}, \mathrm{ViBa}} & \mathbf{C}_{\mathrm{SF}, \mathrm{str}} & \mathbf{C}_{\mathrm{F}}+\mathbf{C}_{\mathrm{LPM}}
\end{array}\right] \dot{\mathbf{u}}(\mathrm{t}) } \\
+\left[\begin{array}{ccc}
\mathbf{K}_{\mathrm{ViBa}} & \mathbf{0} & \mathbf{K}_{\mathrm{ViBa}, \mathrm{SF}} \\
\mathbf{0} & \mathbf{K}_{\mathrm{str}} & \mathbf{K}_{\mathrm{str}, \mathrm{SF}} \\
\mathbf{K}_{\mathrm{SF}, \mathrm{ViBa}} & \mathbf{K}_{\mathrm{SF}, \mathrm{str}} & \mathbf{K}_{\mathrm{F}}+\mathbf{K}_{\mathrm{LPM}}
\end{array}\right] \mathbf{u}(\mathrm{t})=\mathbf{f}(\mathrm{t})
\end{aligned}
$$

Afterwards, the Craig-Bampton method is applied. The method consists of partitioning the global system into two or more subdomains by holding the boundary conditions fixed and then combining the fixed base modal shapes with the constraint modes of the common interface by means of a modal synthesis. Hereafter, the formulation is specialized to the specific case involved in the paper and depicted in Figure 1 even though it can be easily generalized to include more structures and ViBa devices. The physical coordinates $\mathbf{u}$, are transformed to a hybrid set of physical coordinates at the boundary $\mathbf{u}_{\mathrm{SF}}$, and modal coordinates at the interior points of the structure, $\mathbf{q}_{\mathrm{str}}$, and of the ViBa, $\mathbf{q}_{\text {ViBa }}$. By truncating the modal coordinates to smaller sets, let indicate with $\boldsymbol{\Psi}_{[\mathrm{pxi}]}^{\mathrm{ViBa}}$ and $\boldsymbol{\Psi}_{[\mathrm{q} \text { xl }]}^{\mathrm{str}}$ the [p $\mathrm{x}$ i] and [q x l]-matrices of the dynamic modal shapes obtained by conventional eigenvalues problem and with $\boldsymbol{\phi}_{[\mathrm{pxr}]}^{\mathrm{ViBa}}$ and $\boldsymbol{\phi}_{[\mathrm{q} \times r]}^{\mathrm{str}}$ the [p x r] and [q x r] matrices of interface modal shapes of ViBa and structure, respectively. The constraint modes or interface modes $\boldsymbol{\phi}$ relate the rigid body static unit displacements at the interface $\mathbf{u}_{\mathrm{SF}}$ to the physical displacements of the elastic degrees of freedom $\mathbf{u}$. Furthermore, in the case of rigid foundation, the number of constraint modes contained in $\boldsymbol{\phi}$ is sensibly 
reduced according to the number of degree of freedoms of the foundation master nodes. Therefore, the generalized coordinate $[\mathrm{m}=\mathrm{i}+\mathrm{l}+\mathrm{r}]$-vector $\mathbf{q}$ is given by:

$$
\mathbf{q}^{\mathrm{T}}=\left[\mathbf{q}_{\mathrm{ViBa}} \mathbf{q}_{\mathrm{str}} \mathbf{u}_{\mathrm{SF}}\right]
$$

And it is related to the physical coordinates $\mathbf{u}$, by means of the following relation:

$$
\left[\begin{array}{c}
\mathbf{u}_{\mathrm{ViBa}} \\
\mathbf{u}_{\mathrm{str}} \\
\mathbf{u}_{\mathrm{SF}}
\end{array}\right]=\mathbf{P}\left[\begin{array}{c}
\mathbf{q}_{\mathrm{ViBa}} \\
\mathbf{q}_{\mathrm{str}} \\
\mathbf{u}_{\mathrm{SF}}
\end{array}\right]
$$

where $\mathbf{P}$ is the reduced Craig-Bampton transformation matrix:

$$
\mathbf{P}_{[\mathrm{nxm}]}=\left[\begin{array}{ccc}
\boldsymbol{\Psi}_{[\mathrm{pxi}]}^{\mathrm{ViBa}} & \mathbf{0}_{[\mathrm{pxl}]} & \boldsymbol{\phi}_{[\mathrm{pxr}]}^{\mathrm{ViBa}} \\
\mathbf{0}_{[\mathrm{qxi}]} & \boldsymbol{\Psi}_{[\mathrm{qxl}]}^{\text {str }} & \boldsymbol{\phi}_{[\mathrm{qxr}]}^{\text {str }} \\
\mathbf{0}_{[\mathrm{rxi}]} & \mathbf{0}_{[\mathrm{rxl}]} & \mathbf{I}_{[\mathrm{rxr}]}
\end{array}\right]
$$

Note that the physical displacements of the interior points are computed by

$$
\left\{\begin{array}{c}
\mathbf{u}_{\mathrm{ViBa}}=\boldsymbol{\psi}^{\mathrm{ViBa}} \mathbf{q}_{\mathrm{ViBa}}+\boldsymbol{\phi}^{\mathrm{ViBa}} \mathbf{u}_{\mathrm{SF}} \\
\mathbf{u}_{\mathrm{str}}=\boldsymbol{\Psi}^{\mathrm{str}} \mathbf{q}_{\mathrm{str}}+\boldsymbol{\phi}^{\mathrm{str}} \mathbf{u}_{\mathrm{SF}}
\end{array}\right.
$$

The projection of the dynamic governing Eq. (7) over the base $\mathbf{P}$, yields to the Craig-Bampton equation of motion of the reduced model in the time domain:

$$
\mathbf{P}^{\mathrm{T}} \mathbf{M P \ddot { q }}(\mathrm{t})+\mathbf{P}^{\mathrm{T}} \mathbf{C P} \dot{\mathbf{q}}(\mathrm{t})+\mathbf{P}^{\mathrm{T}} \mathbf{K P q}(\mathrm{t})=\mathbf{P}^{\mathrm{T}} \mathbf{f}(\mathrm{t})
$$

where the size of each reduced matrices $\mathbf{P}^{\mathrm{T}} \mathbf{M P}, \mathbf{P}^{\mathrm{T}} \mathbf{C P}$, and $\mathbf{P}^{\mathrm{T}} \mathbf{K P}$ is $[\mathrm{mx} \mathrm{m}]$ with $\mathrm{m}<<\mathrm{n}$.

Furthermore, in case of rigid foundation, the number of constraint modes is sensibly reduced to $\mathrm{r}=$ 12 corresponding to the degree of freedoms of the master nodes of the two foundations involved in this paper. Remarkably, it has to be emphasized that the use of frequency-independent LPM allows the transformation of hysteretic damping model for the soil strictly valid only in the frequency domain to a viscous damping model used in the conventional time-history analysis. 


\section{Dynamic response sensitivity for deterministic load}

In this section, the sensitivity of the reduced model obtained by projection of the global system onto the Craig-Bampton base is investigated. The first-order sensitivity of the deterministic response of the system is evaluated by means of the approach presented in Cacciola et al. (2005). The standard sensitivity analysis entails the evaluation of the derivative of the response of the system with respect to significant system parameters, collected in the vector $\boldsymbol{\alpha}$. According to the dynamic modification approach (Muscolino 1996), the significant system parameters are defined in the neighbourhood of prefixed values, called nominal parameter values. Therefore, by denoting with $\boldsymbol{\alpha}_{0}$ the vector of the nominal parameters, the vector $\boldsymbol{\alpha}$ of the actual values is estimated as $\boldsymbol{\alpha}=\boldsymbol{\alpha}_{0}+\Delta \boldsymbol{\alpha}$, where $\Delta \boldsymbol{\alpha}$ lists the small parameter variations from the nominal values. Due to the dependence of the system on the actual values of $\boldsymbol{\alpha}$, let the vector of state variables $\mathbf{z}(\boldsymbol{\alpha}, \mathrm{t})$ of order [ $2 \mathrm{~m} \times 1$ ] be introduced in the form:

$$
\mathbf{z}(\boldsymbol{\alpha}, \mathrm{t})=\left[\begin{array}{c}
\mathbf{q}(\boldsymbol{\alpha}, \mathrm{t}) \\
\dot{\mathbf{q}}(\boldsymbol{\alpha}, \mathrm{t})
\end{array}\right]
$$

Therefore, from Eq. (13), the governing equations of motion in state variable modal space for the reduced model, are derived as follows:

$$
\dot{\mathbf{z}}(\boldsymbol{\alpha}, \mathrm{t})=\mathbf{D}(\boldsymbol{\alpha}) \mathbf{z}(\boldsymbol{\alpha}, \mathrm{t})+\mathbf{V}(\boldsymbol{\alpha}) \mathbf{f}(\boldsymbol{\alpha}, \mathrm{t})
$$

where:

$$
\mathbf{D}(\boldsymbol{\alpha})=\left[\begin{array}{cc}
\mathbf{0}_{[\mathrm{mxm}]} & \mathbf{I}_{[\mathrm{mxm}]} \\
-\widetilde{\mathbf{M}}_{\boldsymbol{\alpha}}^{-1} \mathbf{P}_{\boldsymbol{\alpha}_{0}}^{\mathrm{T}} \mathbf{K}(\boldsymbol{\alpha}) \mathbf{P}_{\boldsymbol{\alpha}_{0}} & -\widetilde{\mathbf{M}}_{\boldsymbol{\alpha}}^{-1} \mathbf{P}_{\boldsymbol{\alpha}_{0}}^{\mathrm{T}} \mathbf{C}(\boldsymbol{\alpha}) \mathbf{P}_{\boldsymbol{\alpha}_{0}}
\end{array}\right]
$$

with $\widetilde{\mathbf{M}}_{\boldsymbol{\alpha}}=\mathbf{P}_{\boldsymbol{\alpha}_{0}}^{\mathrm{T}} \mathbf{M}(\boldsymbol{\alpha}) \mathbf{P}_{\boldsymbol{\alpha}_{0}}$, and

$$
\mathbf{V}(\boldsymbol{\alpha})=\left[\begin{array}{c}
\mathbf{0}_{[\mathrm{mxm}]} \\
\widetilde{\mathbf{M}}_{\boldsymbol{\alpha}}^{-1} \mathbf{P}_{\boldsymbol{\alpha}_{0}}^{\mathrm{T}}
\end{array}\right]
$$

The force vector $\mathbf{f}(\boldsymbol{\alpha}, \mathrm{t})$ lists the load applied to the structure and the explicit dependence on $\boldsymbol{\alpha}$ arises from the seismic force $\mathbf{f}_{\mathrm{SF}}$ defined at the soil-structure interface level: 


$$
\mathbf{f}_{\mathrm{SF}}(\boldsymbol{\alpha}, \mathrm{t})=\int_{-\infty}^{\infty} \mathbf{f}_{\mathrm{s}}(\boldsymbol{\alpha}, \mathrm{t}-\tau) \mathrm{u}_{\mathrm{g}}(\tau) d \tau
$$

where $u_{g}(t)$ is the inverse Fourier transform of the free field motion used in Eq. (4) The reduced Craig-Bampton transformation matrix $\mathbf{P}_{\boldsymbol{\alpha}_{0}}$ is evaluated according to Eq. (11) in correspondence of the nominal values $\boldsymbol{\alpha}_{0}$.

It is worth mentioning that Eq. (15) represents the equation of motion in state variable modal space for the reduced model. The solution of Eq. (15) in integral form can be written as:

$$
\mathbf{z}(t)=\boldsymbol{\theta}_{0}\left(\mathrm{t}-\mathrm{t}_{0}\right) \mathbf{z}\left(\mathrm{t}_{0}\right)+\int_{\mathbf{t}_{0}}^{\mathrm{t}} \boldsymbol{\theta}_{0}(\mathrm{t}-\tau) \mathbf{V}_{0} \mathbf{f}(\tau) \mathrm{d} \tau
$$

where $\boldsymbol{\theta}_{0}(\mathrm{t})$ is the transition matrix given by :

$$
\boldsymbol{\theta}_{0}(\mathrm{t})=\exp (\mathbf{D t})
$$

Once the modal response is determined, the conventional response in nodal space in terms of displacements is obtained as follows:

$$
\mathbf{u}(\mathrm{t})=\mathbf{P q}(\mathrm{t})=\mathbf{P}\left[\mathbf{I}_{[\mathrm{mxm}]} \quad \mathbf{0}_{[\mathrm{mxm}]}\right] \mathbf{z}(\mathrm{t})
$$

The evolution of the deterministic sensitivity in the neighbourhood of nominal values $\boldsymbol{\alpha}_{0}$, is obtained by differentiating Eq. (15) with respect to ith significant parameter $\alpha_{i}$; therefore, it is governed by the following first-order differential equations:

$\dot{\mathbf{s}}_{\mathrm{z}, \mathrm{i}}\left(\boldsymbol{\alpha}_{0}, \mathrm{t}\right)=\mathbf{D}\left(\boldsymbol{\alpha}_{0}\right) \mathbf{s}_{\mathrm{z}, \mathrm{i}}\left(\boldsymbol{\alpha}_{0}, \mathrm{t}\right)+\mathbf{A}_{\mathrm{i}}\left(\boldsymbol{\alpha}_{0}\right) \mathbf{z}\left(\boldsymbol{\alpha}_{0}, \mathrm{t}\right)+\mathbf{B}_{\mathrm{i}}\left(\boldsymbol{\alpha}_{0}\right) \mathbf{f}\left(\boldsymbol{\alpha}_{0}, \mathrm{t}\right)+\mathbf{V}\left(\boldsymbol{\alpha}_{0}\right) \mathbf{g}_{\mathrm{i}}\left(\boldsymbol{\alpha}_{0}, \mathrm{t}\right)$

where by denoting with [.] $]^{\mathrm{I}}$ the derivative of the matrices with respect to ith significant parameter $\alpha_{\mathrm{i}}$ :

$$
\mathbf{A}_{\mathrm{i}}\left(\boldsymbol{\alpha}_{0}\right)=\left.\frac{\partial}{\partial \alpha_{\mathrm{i}}} \mathbf{D}(\boldsymbol{\alpha})\right|_{\boldsymbol{\alpha}=\boldsymbol{\alpha}_{0}}=\left[\begin{array}{cc}
\mathbf{0} & \mathbf{0} \\
-\mathbf{a}_{1, \mathrm{i}}\left(\boldsymbol{\alpha}_{0}\right) & -\mathbf{a}_{2, \mathrm{i}}\left(\boldsymbol{\alpha}_{0}\right)
\end{array}\right]
$$

in which the quantity $\mathbf{a}_{1, \mathrm{i}}\left(\boldsymbol{\alpha}_{0}\right)$ is 


$$
\mathbf{a}_{1, \mathrm{i}}=-\widetilde{\mathbf{M}}_{\boldsymbol{\alpha}_{0}}^{-1} \widetilde{\mathbf{M}}_{\boldsymbol{\alpha}_{0}, \mathrm{i}}^{\mathrm{I}} \widetilde{\mathbf{M}}_{\boldsymbol{\alpha}_{0}}^{-1} \mathbf{P}_{\boldsymbol{\alpha}_{0}}^{\mathrm{T}} \mathbf{K} \mathbf{P}_{\boldsymbol{\alpha}_{0}}+\widetilde{\mathbf{M}}_{\boldsymbol{\alpha}_{0}}^{-1} \mathbf{P}_{\boldsymbol{\alpha}_{0}}^{\mathrm{T}} \mathbf{K}_{\mathrm{i}}^{\mathrm{I}} \mathbf{P}_{\boldsymbol{\alpha}_{\mathbf{0}}}
$$

and the quantity $\mathbf{a}_{2, \mathrm{i}}\left(\boldsymbol{\alpha}_{0}\right)$ is

$$
\mathbf{a}_{2, \mathrm{i}}=-\widetilde{\mathbf{M}}_{\boldsymbol{\alpha}_{0}}^{-1} \widetilde{\mathbf{M}}_{\boldsymbol{\alpha}_{0}, \mathrm{i}}^{\mathrm{I}} \widetilde{\mathbf{M}}_{\boldsymbol{\alpha}_{0}}^{-1} \mathbf{P}_{\boldsymbol{\alpha}_{0}}^{\mathrm{T}} \mathbf{C} \mathbf{P}_{\boldsymbol{\alpha}_{0}}+\widetilde{\mathbf{M}}_{\boldsymbol{\alpha}_{0}}^{-1} \mathbf{P}_{\boldsymbol{\alpha}_{0}}^{\mathrm{T}} \mathbf{C}_{\mathrm{i}}^{\mathrm{I}} \mathbf{P}_{\boldsymbol{\alpha}_{0}}
$$

In the above equations, the following position $\widetilde{\mathbf{M}}_{\boldsymbol{\alpha}_{0}}=\mathbf{P}_{\boldsymbol{\alpha}_{0}}^{\mathrm{T}} \mathbf{M}\left(\boldsymbol{\alpha}_{0}\right) \mathbf{P}_{\boldsymbol{\alpha}_{0}}$ is used and the arguments are omitted. Finally, $\mathbf{B}_{\mathbf{i}}\left(\boldsymbol{\alpha}_{0}\right)$ is:

$$
\mathbf{B}_{\mathrm{i}}\left(\boldsymbol{\alpha}_{0}\right)=\left[\begin{array}{c}
\mathbf{0}_{[\mathrm{mxm}]} \\
-\widetilde{\mathbf{M}}_{\boldsymbol{\alpha}_{0}}^{-1} \widetilde{\mathbf{M}}_{\boldsymbol{\alpha}_{0}, \mathrm{i}}^{\mathrm{I}} \widetilde{\mathbf{M}}_{\boldsymbol{\alpha}_{0}}^{-1} \mathbf{P}_{\boldsymbol{\alpha}_{0}}^{\mathrm{T}}
\end{array}\right]
$$

and

$$
\mathbf{g}_{\mathrm{i}}\left(\boldsymbol{\alpha}_{\mathbf{0}}, \mathrm{t}\right)=\left.\frac{\partial \mathbf{f}(\boldsymbol{\alpha}, \mathrm{t})}{\partial \alpha_{\mathrm{i}}}\right|_{\boldsymbol{\alpha}=\boldsymbol{\alpha}_{0}}
$$

Therefore, the sensitivity of the response of the non-classically damped system is obtained by numerically integrating the sensitivity equation of Eq. (19) by means of the same numerical procedure adopted to solve the governing equation of the motion Eq. (15) due to their similar mathematical structure once the pseudo-force is set as:

$$
\overline{\mathbf{F}}\left(\boldsymbol{\alpha}_{0}, t\right)=\mathbf{A}_{\mathrm{i}}\left(\boldsymbol{\alpha}_{0}\right) \mathbf{z}\left(\boldsymbol{\alpha}_{0}, \mathrm{t}\right)+\mathbf{B}_{\mathrm{i}}\left(\boldsymbol{\alpha}_{0}\right) \mathbf{f}\left(\boldsymbol{\alpha}_{0}, \mathrm{t}\right)+\mathbf{V}\left(\boldsymbol{\alpha}_{0}\right) \mathbf{g}_{\mathrm{i}}\left(\boldsymbol{\alpha}_{0}, \mathrm{t}\right)
$$

Finally, the sensitivity response of the displacements in the nodal space is straightforward evaluated as follows:

$$
\mathbf{s}_{\mathrm{u}, \mathrm{i}}\left(\boldsymbol{\alpha}_{\mathbf{0}}, \mathrm{t}\right)=\mathbf{P}_{\boldsymbol{\alpha}_{0}}\left[\mathbf{I}_{[\mathrm{mxm}]} \quad \mathbf{0}_{[\mathrm{mxm}]}\right] \mathbf{s}_{\mathrm{z}, \mathrm{i}}\left(\boldsymbol{\alpha}_{0}, \mathrm{t}\right)
$$

This method allows the evaluation of the sensitivity response according to the dynamic modification approach in which the Craig-Bampton transformation matrix is determined at the nominal values $\boldsymbol{\alpha}_{0}$ 
and not at the exact values $\boldsymbol{\alpha}$. Therefore, the following approximation $\mathbf{P}(\boldsymbol{\alpha}) \cong \mathbf{P}_{\boldsymbol{\alpha}_{0}}$ is done; the error in the results is small if the modal shapes is not sensibly affected from the parameters $\boldsymbol{\alpha}$.

\section{Dynamic response sensitivity for stochastic load process}

In this section, the sensitivity of the response for a system subjected to non-stationary zero mean Gaussian stochastic load process is accomplished. Consider the filtered multi-variate process fully defined by the knowledge of its power spectral density matrix:

$$
\mathbf{S}_{\mathrm{FF}}(\omega, \mathrm{t})=\left[\begin{array}{c}
\mathbf{0} \\
\mathbf{0} \\
\mathbf{f}_{\mathrm{S}}(\omega)
\end{array}\right]\left[\begin{array}{lll}
\mathbf{0} & \mathbf{0} & \mathbf{f}_{\mathrm{s}}^{*}(\omega)
\end{array}\right] \mathrm{S}_{\mathrm{g}}(\omega, \mathrm{t})
$$

where $[\cdot]^{*}$ denotes the conjugate transpose operator, and $S_{g}(\omega, t)$ is the power spectral density function of the free field ground motion. The sensitivity of the stochastic response is evaluated in terms of variation of the statistical state-space second-order moment; therefore a direct linear stochastic differential equation of motion is determined.

The second-order moment of the response in the state variable space can be obtained as:

$$
\mathbf{m}_{\mathbf{Z}}^{(2)}(\boldsymbol{\alpha}, \mathrm{t})=\mathrm{E}[\mathbf{Z}(\boldsymbol{\alpha}, \mathrm{t}) \otimes \mathbf{Z}(\boldsymbol{\alpha}, \mathrm{t})]=\operatorname{Vec}\left\{\mathrm{E}\left[\mathbf{Z}(\boldsymbol{\alpha}, \mathrm{t}) \mathbf{Z}^{\mathrm{T}}(\boldsymbol{\alpha}, \mathrm{t})\right]\right\}
$$

where E[·] denotes mathematical expectation, the symbol $\otimes$ is the Kronecker product and $\mathbf{m}_{\mathbf{Z}}^{(2)}(\boldsymbol{\alpha}, \mathrm{t})$ is the vectorialized form, $\operatorname{Vec}\{\cdot\}$, of the cross-covariance matrix defined for the dynamically modified system.

By differentiating Eq. (31) with respect the time, the evolution of the second-order statistical moments of the response in the state variable space is determined after simple algebra:

$$
\dot{\mathbf{m}}_{\mathrm{Z}}^{(2)}(\boldsymbol{\alpha}, \mathrm{t})=\mathbf{D}_{\mathbf{2}}(\boldsymbol{\alpha}) \mathbf{m}_{\mathbf{Z}}^{(2)}(\boldsymbol{\alpha}, \mathrm{t})+\mathbf{F}_{2}(\boldsymbol{\alpha}, \mathrm{t})
$$

where:

$$
\mathbf{F}_{2}(\boldsymbol{\alpha}, \mathrm{t})=\left[\mathbf{I}_{2 \mathrm{~m}} \otimes \mathbf{V}(\boldsymbol{\alpha})\right] \mathrm{E}[\mathbf{Z}(\boldsymbol{\alpha}, \mathrm{t}) \otimes \mathbf{f}(\boldsymbol{\alpha}, \mathrm{t})]+\left[\mathbf{V}(\boldsymbol{\alpha}) \otimes \mathbf{I}_{2 \mathrm{~m}}\right] \mathrm{E}[\mathbf{f}(\boldsymbol{\alpha}, \mathrm{t}) \otimes \mathbf{Z}(\boldsymbol{\alpha}, \mathrm{t})]
$$


in which $\mathbf{I}_{2 \mathrm{~m}}=\mathbf{I}_{[2 \mathrm{mx} 2 \mathrm{~m}]}$ and, by using the Kronecker sum $\oplus$ :

$$
\mathbf{D}_{2}(\boldsymbol{\alpha})=\mathbf{D}(\boldsymbol{\alpha}) \oplus \mathbf{D}(\boldsymbol{\alpha})
$$

Finally, the differential equations governing the evolution of the sensitivity of the second-order statistical moments of the response in the state variable space is determined by differentiating Eq. (31) with respect to ith significant parameter $\alpha_{i}$ :

$$
\dot{\mathbf{s}}_{\mathrm{Z}, \mathrm{i}}^{(2)}\left(\boldsymbol{\alpha}_{0}, \mathrm{t}\right)=\mathbf{D}_{2}\left(\boldsymbol{\alpha}_{0}\right) \mathbf{s}_{\mathrm{Z}, \mathrm{i}}^{(2)}\left(\boldsymbol{\alpha}_{0}, \mathrm{t}\right)+\overline{\mathbf{F}}_{2}\left(\boldsymbol{\alpha}_{0}, \mathrm{t}\right)
$$

where the pseudo-force $\overline{\mathbf{F}}_{2}\left(\boldsymbol{\alpha}_{0}, \mathrm{t}\right)$ lists the cross-correlation terms as follows:

$$
\begin{gathered}
\overline{\mathbf{F}}_{2}\left(\boldsymbol{\alpha}_{\mathbf{0}}, \mathrm{t}\right)=\mathbf{A}_{2, \mathrm{i}}\left(\boldsymbol{\alpha}_{0}\right) \mathbf{m}_{\mathrm{Z}}^{(2)}\left(\boldsymbol{\alpha}_{0}, \mathrm{t}\right)+\left[\mathbf{I}_{2 \mathrm{~m}} \otimes \mathbf{B}\left(\boldsymbol{\alpha}_{0}\right)\right] \mathrm{E}\left[\mathbf{Z}\left(\boldsymbol{\alpha}_{0}, \mathrm{t}\right) \otimes \mathbf{f}\left(\boldsymbol{\alpha}_{0}, \mathrm{t}\right)\right]+ \\
{\left[\mathbf{B}\left(\boldsymbol{\alpha}_{0}\right) \otimes \mathbf{I}_{2 \mathrm{~m}}\right] \mathrm{E}\left[\mathbf{f}\left(\boldsymbol{\alpha}_{0}, \mathrm{t}\right) \otimes \mathbf{Z}(\boldsymbol{\alpha}, \mathrm{t})\right]+\left[\mathbf{I}_{2 \mathrm{~m}} \otimes \mathbf{V}\left(\boldsymbol{\alpha}_{0}\right)\right]\left\{\mathrm{E}\left[\mathbf{s}_{\mathrm{z}, \mathrm{i}}\left(\boldsymbol{\alpha}_{0}, \mathrm{t}\right) \otimes \mathbf{f}\left(\boldsymbol{\alpha}_{0}, \mathrm{t}\right)\right]+\right.} \\
\left.\mathrm{E}\left[\mathbf{Z}\left(\boldsymbol{\alpha}_{0}, \mathrm{t}\right) \otimes \mathbf{g}_{\mathrm{i}}\left(\boldsymbol{\alpha}_{0}, \mathrm{t}\right)\right]\right\}+\left[\mathbf{V}\left(\boldsymbol{\alpha}_{0}\right) \otimes \mathbf{I}_{2 \mathrm{~m}}\right]\left\{\mathrm{E}\left[\mathbf{f}\left(\boldsymbol{\alpha}_{0}, \mathrm{t}\right) \otimes \mathbf{s}_{\mathrm{z}, \mathrm{i}}\left(\boldsymbol{\alpha}_{0}, \mathrm{t}\right)\right]+\mathrm{E}\left[\mathbf{g}_{\mathrm{i}}\left(\boldsymbol{\alpha}_{0}, \mathrm{t}\right) \otimes \mathbf{Z}\left(\boldsymbol{\alpha}_{0}, \mathrm{t}\right)\right]\right\}(36)
\end{gathered}
$$

with

$$
\mathbf{A}_{2, \mathrm{i}}\left(\boldsymbol{\alpha}_{0}\right)=\mathbf{A}_{\mathrm{i}}\left(\boldsymbol{\alpha}_{0}\right) \oplus \mathbf{A}_{\mathrm{i}}\left(\boldsymbol{\alpha}_{0}\right)
$$

Consider the input as non-stationary zero-mean Gaussian stochastic process fully defined by the power spectral density (PSD) matrix function $\mathbf{S}_{\mathrm{FF}}(\boldsymbol{\alpha}, \omega, \mathrm{t})$ as defined in Eq. (30). After simple algebra, the stochastic averages $E[\mathbf{Z}(\boldsymbol{\alpha}, t) \otimes \mathbf{f}(\boldsymbol{\alpha}, t)]$ and $E[\mathbf{f}(\boldsymbol{\alpha}, t) \otimes \mathbf{Z}(\boldsymbol{\alpha}, t)]$ of Eq. (36) are obtained as follows:

$$
\mathrm{E}[\mathbf{Z}(\boldsymbol{\alpha}, \mathrm{t}) \otimes \mathbf{f}(\boldsymbol{\alpha}, \mathrm{t})] \cong \int_{-\infty}^{\infty}\left[\mathbf{H}_{0}^{*}(\omega, \boldsymbol{\alpha}) \otimes \mathbf{I}_{\mathrm{m}}\right] \operatorname{Vec}\left\{\mathbf{S}_{\mathrm{FF}}(\boldsymbol{\alpha}, \omega, \mathrm{t})\right\} \mathrm{d} \omega
$$

and

$$
\mathrm{E}[\mathbf{f}(\boldsymbol{\alpha}, \mathrm{t}) \otimes \mathbf{Z}(\boldsymbol{\alpha}, \mathrm{t})] \cong \int_{-\infty}^{\infty}\left[\mathbf{I}_{\mathrm{m}} \otimes \mathbf{H}_{0}(\omega, \boldsymbol{\alpha})\right] \operatorname{Vec}\left\{\mathbf{S}_{\mathrm{FF}}(\boldsymbol{\alpha}, \omega, \mathrm{t})\right\} \mathrm{d} \omega
$$

in which $\mathbf{I}_{\mathrm{m}}=\mathbf{I}_{[\mathrm{mxm}]}$. The matrix $\mathbf{H}_{0}(\omega, \boldsymbol{\alpha})$ is the transfer function matrix of the system in the frequency domain: 


$$
\mathbf{H}_{0}(\omega, \boldsymbol{\alpha})=\left[\mathrm{i} \omega \mathbf{I}_{2 \mathrm{~m}}-\mathbf{D}(\boldsymbol{\alpha})\right]^{-1} \mathbf{V}(\boldsymbol{\alpha})
$$

It should be emphasized that the relations in Eq. (38) and in Eq. (39) are exact only in case of stationary input, that it $S_{F F}(\omega, t)=S_{F F}(\omega)$. For weakly nonstationary systems they provide a satisfactory approximation. For a rigorous evaluation of the cross statistics the approach described in Di Paola and Petrucci (1990) and Cacciola and Muscolino (2011) herein omitted for simplicity sake can be adopted alternatively.

Moreover, by differentiating Eq. (38) and Eq. (39) with respect to the sensitivity parameter $\boldsymbol{\alpha}$, the cross-correlation functions $\partial \mathrm{E}[\mathbf{Z}(\boldsymbol{\alpha}, \mathrm{t}) \otimes \mathbf{f}(\boldsymbol{\alpha}, \mathrm{t})] / \partial \alpha_{\mathrm{i}}=\mathrm{E}\left[\mathbf{s}_{\mathrm{z}, \mathrm{i}}(\boldsymbol{\alpha}, \mathrm{t}) \otimes \mathbf{f}(\boldsymbol{\alpha}, \mathrm{t})\right]+\mathrm{E}\left[\mathbf{Z}(\boldsymbol{\alpha}, \mathrm{t}) \otimes \mathbf{g}_{\mathrm{i}}(\boldsymbol{\alpha}, \mathrm{t})\right]$ and $\partial \mathrm{E}[\mathbf{f}(\boldsymbol{\alpha}, \mathrm{t}) \otimes \mathbf{Z}(\boldsymbol{\alpha}, \mathrm{t})] / \partial \alpha_{\mathrm{i}}=\mathrm{E}\left[\mathbf{f}(\boldsymbol{\alpha}, \mathrm{t}) \otimes \mathbf{s}_{\mathrm{z}, \mathrm{i}}(\boldsymbol{\alpha}, \mathrm{t})\right]+\mathrm{E}\left[\mathbf{g}_{\mathrm{i}}(\boldsymbol{\alpha}, \mathrm{t}) \otimes \mathbf{Z}(\boldsymbol{\alpha}, \mathrm{t})\right]$ are derived as follows:

$$
\begin{aligned}
\mathrm{E}\left[\mathbf{s}_{\mathrm{z}, \mathrm{i}}(\boldsymbol{\alpha}, \mathrm{t}) \otimes \mathbf{f}\right. & (\boldsymbol{\alpha}, \mathrm{t})] \\
& \cong \int_{-\infty}^{\infty}\left\{\left\{\left[\mathbf{H}_{0}^{*}(\omega, \boldsymbol{\alpha})\right]^{-1} \mathbf{A}_{\mathrm{i}}(\boldsymbol{\alpha})\left[\mathbf{H}_{0}^{*}(\omega, \boldsymbol{\alpha})\right]^{-1}\right.\right. \\
& \left.\left.+\left[\mathbf{H}_{0}^{*}(\omega, \boldsymbol{\alpha})\right]^{-1} \mathbf{B}(\boldsymbol{\alpha})\right\} \otimes \mathbf{I}_{\mathrm{m}}\right\} \operatorname{Vec}\left\{\mathbf{S}_{\mathrm{FF}}(\boldsymbol{\alpha}, \omega, \mathrm{t})\right\} \mathrm{d} \omega
\end{aligned}
$$

and

$$
\mathrm{E}\left[\mathbf{Z}(\boldsymbol{\alpha}, \mathrm{t}) \otimes \mathbf{g}_{\mathrm{i}}(\boldsymbol{\alpha}, \mathrm{t})\right] \cong \int_{-\infty}^{\infty}\left[\mathbf{H}_{0}^{*}(\omega, \boldsymbol{\alpha}) \otimes \mathbf{I}_{\mathrm{m}}\right] \operatorname{Vec}\left\{\partial \mathbf{S}_{\mathrm{FF}}(\boldsymbol{\alpha}, \omega, \mathrm{t}) / \partial \alpha_{\mathrm{i}}\right\} \mathrm{d} \omega
$$

as well as

$$
\begin{aligned}
\mathrm{E}\left[\mathbf{f}(\boldsymbol{\alpha}, \mathrm{t}) \otimes \mathbf{s}_{\mathrm{z}, \mathrm{i}}\right. & (\boldsymbol{\alpha}, \mathrm{t})] \\
& \cong \int_{-\infty}^{\infty}\left\{\mathbf { I } _ { \mathrm { m } } \otimes \left\{\left[\mathbf{H}_{0}(\omega, \boldsymbol{\alpha})\right]^{-1} \mathbf{A}_{\mathrm{i}}(\boldsymbol{\alpha})\left[\mathbf{H}_{0}(\omega, \boldsymbol{\alpha})\right]^{-1}\right.\right. \\
& \left.\left.+\left[\mathbf{H}_{0}(\omega, \boldsymbol{\alpha})\right]^{-1} \mathbf{B}(\boldsymbol{\alpha})\right\}\right\} \operatorname{Vec}\left\{\mathbf{S}_{\mathrm{FF}}(\boldsymbol{\alpha}, \omega, \mathrm{t})\right\} \mathrm{d} \omega
\end{aligned}
$$

$$
\mathrm{E}\left[\mathbf{g}_{\mathrm{i}}(\boldsymbol{\alpha}, \mathrm{t}) \otimes \mathbf{Z}(\boldsymbol{\alpha}, \mathrm{t})\right] \cong \int_{-\infty}^{\infty}\left[\mathbf{I}_{\mathrm{m}} \otimes \mathbf{H}_{0}(\omega, \boldsymbol{\alpha})\right] \operatorname{Vec}\left\{\partial \mathbf{S}_{\mathrm{FF}}(\boldsymbol{\alpha}, \omega, \mathrm{t}) / \partial \alpha_{\mathrm{i}}\right\} \mathrm{d} \omega
$$


By calculating each cross-correlation term, the pseudo-force of Eq. (36) is derived and the sensitivity of the response for a system subjected to zero mean Gaussian quasi-stationary stochastic load process is determined accordingly.

\section{Numerical Application}

In this section, the proposed procedure is applied to investigate the sensitivity of the stochastic response of the model of an Industrial Building protected by the ViBa as depicted in Figure 1. The simple model of a industrial building, described in the report EPRI (2006), is chosen. The relevant dimensions are summarized in Table 1. The structure is founded on 30m-thick soil deposit characterized by shear wave velocity $V_{s}=400 \mathrm{~m} / \mathrm{s}^{2}$ and hysteretic damping $\eta_{s}=0.1$ resting on stiff bedrock with shear wave velocity of $V_{s}=800 \mathrm{~m} / \mathrm{s}^{2}$ and hysteretic damping $\eta_{\text {bed }}=0.05$. The ViBa is externally modelled as a circular embedded foundation characterized by dimensions of the radius and the embedded height equal to half as much as those related to the building as reported in Table 2. Both structures are modelled by concrete shell elements. The internal structure of the ViBa is a single oscillator characterized by the internal mass, $m_{V i B a}$, the stiffness, $k_{V i B a}$, and the damping ratio $\xi_{V i B a}$.

The structure is modelled according to the finite element approach by means of the Code_Aster open source FE-software (2013) whereas the BEM formulation is used to model the soil by means of Miss3D (Clouteau, 2005) ), available with SalomeMeca leading to 25651 DoFs. The sensitivity of the stochastic response of the structure is investigated by means of the procedure proposed in this paper. Firstly, a reduced model is obtained in order to capture the structural behavior by means of the CraigBampton procedure and the approximation of the foundation-soil-foundation model by LPM, which consists of only 6 DoFs. According to the proposed procedure, the reduced global model is obtained once the vector of generalized coordinates $\mathbf{q}^{\mathrm{T}}=\left[\mathrm{q}_{\mathrm{ViBa}}^{\mathrm{x}}, \mathrm{q}_{\mathrm{str}}^{\mathrm{x}}, \mathrm{u}_{\mathrm{SF}, \mathrm{str}}^{\mathrm{x}}, \mathrm{u}_{\mathrm{SF}, \mathrm{str}}^{\theta}, \mathrm{u}_{\mathrm{SF}, \mathrm{ViBa}}^{\mathrm{x}}, \mathrm{u}_{\mathrm{SF}, \mathrm{ViBa}}^{\theta}\right]$ is 
determined, where $\mathrm{q}_{\mathrm{ViBa}}^{\mathrm{x}}$ and $\mathrm{q}_{\mathrm{str}}^{\mathrm{x}}$ are the first generalized coordinates in $\mathrm{x}$-direction of the ViBa and structure, respectively; $u_{\mathrm{SF}, \mathrm{str}}^{\mathrm{x}} \quad \mathrm{u}_{\mathrm{SF}, \mathrm{str}}^{\theta} \quad \mathrm{u}_{\mathrm{SF}, \mathrm{ViBa}}^{\mathrm{X}} \quad \mathrm{u}_{\mathrm{SF}, \mathrm{ViBa}}^{\theta}$ are the $\mathrm{X}$-and $\theta$ - directions related to the structure and ViBa, respectively.

In order to formulate the model in the time domain, a lumped parameter model is determined for approximating each component of the dynamic stiffness matrix $\mathbf{K}_{\text {dyn }}(\omega)$ by frequency-independent parameters obtained by linear least squares as depicted in Figures $2-3$. As shown in the Figures the frequency-independent parameters provide an excellent approximation of each respective component of the dynamic stiffness matrix in the range 0-5 Hz. Also, the LPM used for the real part of the foundations coupling impedances (see Figure 4 ), indicated by $K_{d y n}^{i, j}(\omega)$ with $i \neq j$, is obtained by calibrating the elastic spring at the first fundamental frequency of the industrial building in the coupled case, i.e. $3.65 \mathrm{~Hz}$. Ground motion acceleration $\ddot{u}_{\mathrm{g}}(\mathrm{t})$ is modelled as a broadband uniformly modulated process $S_{g}(\omega, t)=\varphi(t) S_{w}$ with the cut-off frequency of $5 \mathrm{~Hz}, S_{w}=10^{-6} \mathrm{~m}^{2} / \mathrm{s}^{3}$ and modulating function $\varphi(\mathrm{t})$ given by:

$$
\varphi(t)=12.21\left(\mathrm{e}^{-0.4 \mathrm{t}}-\mathrm{e}^{-0.5 \mathrm{t}}\right)
$$

Monte Carlo Simulation (MCS) is performed by generation of one hundred samples from the adopted input process for both the reduced model and the exact reference model.

Results are focused on the seismic response of the Industrial Building recorded at the top of the dome. For illustrative purpose figure 5 show two responses in acceleration achieved from the analysis of the LPM model undergoing two randomly-selected ground motions and compared with the responses obtained by the FEM model showing an excellent agreement. Moreover, in Figure 6 the power spectral density functions obtained by averaging out the results of the MCS for both models, illustrate the overall validity of the LPM model. 
Sensitivity analysis is performed by the procedure proposed in this paper. The mass $\mathrm{m}_{\mathrm{ViBa}}$ of the $\mathrm{ViBa}$ is initially assigned as $3.1 \times 10^{7} \mathrm{~kg}$ corresponding to the $55 \%$ of the mass of the Reactor Building. As a difference from the other two structural parameters the mass $m_{V i B a}$, is not determined by an optimization procedure but it is assigned by the designer taking into consideration further technical issues such as the bearing capacity of the soil. Furthermore it can be measured a posteriori so to use an accurate value for the design of the internal stiffness, $\mathrm{k}_{\mathrm{ViBa}}$, as well as the damping ratio $\xi_{\mathrm{ViBa}}$. As the mass $\mathrm{m}_{\mathrm{ViBa}}$ is assigned, the optimization design procedure entails only two design parameters. Therefore, the pertinent vector listing the ViBa design sensitivity parameters is: $\boldsymbol{\alpha}=$ $\left[\mathrm{k}_{\mathrm{ViBa}}, \xi_{\mathrm{ViBa}}\right]$. The values of the vector of the nominal parameters, representing the optimal values that minimize the stochastic response, are determined following the procedure proposed in Cacciola and Tombari (2015) and Cacciola et al. (2015), namely:

$$
\boldsymbol{\alpha}_{0}=[1.8634 \mathrm{E}+10 \mathrm{~N} / \mathrm{m} \quad 0.01]
$$

It is worth emphasized that the sensitivity parameters vector, $\boldsymbol{\alpha}$, can be extended to additional system parameters of engineering interest.

In order to show the accuracy of the proposed procedure to determine the sensitivity of the stochastic response of the coupled system in Figure 7a-b the curves of the sensitivity of the second-order moment $\mathrm{s}_{\mathrm{u}, \alpha}^{(2)}(\mathrm{t})$ of the nodal displacement at the top of the structure with respect to the variation of the $\mathrm{ViBa}$ stiffness $\mathrm{k}_{\mathrm{ViBa}}$ and damping $\xi_{\mathrm{ViBa}}$ determined through Eq. (35) are compared with the results obtained by MCS of the FEM model. The good matching between them point out the satisfactory overall accuracy of the proposed method.

For design purpose the non-dimensional sensitivity $\mathrm{s}_{\mathrm{n}}^{(2)}(\mathrm{t})$ proposed in Cacciola et al (2005) given by the following equation 


$$
s_{n, u, \alpha}^{(2)}(t)=\frac{s_{u, \alpha}^{(2)}(t)}{m_{u}^{(2)}(t)} \alpha_{0}
$$

is furthermore determined and depicted in Figure 8. Specifically, the non-dimensional sensitivity $s_{n}^{(2)}(t)$ expresses the amplification of the variation of the second-order moment of the response due to a small change in the pertinent nominal parameters. From the analysis of Figures 8, it is worth noting that the structural response is sensible to a variation of the optimal parameter of stiffness $\mathrm{k}_{\mathrm{ViBa}}$ (Figure 8a) while the response is not significantly affected by a small e variation of the damping $\xi_{\text {ViBa }}$, as shown in Figure 8b.

Sensitivity analysis is furthermore performed in order to evaluate the effects on the stochastic response of the structure due to small variations in the parameters of the LPM used to simulate the structure-soil-structure interaction. This uncertainty derives from the dispersion of the soil mechanical characteristics as well as from uncertainties in the modelling itself. Figure 9a-b shows the sensitivity curves with respect to the $\mathrm{x}$-component of the LPM and to the rotational ry-conponent (impedances depicted in Figure 3), respectively. For comparison purpose, MCS is carried out in the reduced model with 1000 generated samples. Finally, non-dimensional sensitivity are reported in Figure 10. A relevant sensitivity of the seismic response to the rotational component of the LPM is observed .

\section{Concluding Remarks}

A method for the evaluation of the sensitivity of the stochastic response of structures coupled with the novel Vibrating Barrier device is presented. As the coupling involves structure-soil-structure interaction, the traditional hysteretic model is adopted for modelling soil damping. The method therefore requires the following steps: i) evaluation of an equivalent lumped parameter model to move from the frequency domain to the time domain; ii) definition of a reduced model by applying the CraigBampton procedure; iii) evaluation of the response sensitivity in the time domain in a reduce subspace 
and further evaluation of the sensitivity of the nodal response through a back transformation. The procedure proposed in this paper has been applied to investigate the stochastic response of an Industrial Building coupled with the Vibrating Barrier (ViBa) device.

The effectiveness of the procedure adopted for the sensitivity analysis and for the approximation of the soil with lumped parameter models has been proved by positive comparison with Monte Carlo Finally, the response sensitivity has been evaluated with respect to the main design parameters of the ViBa and the parameters of the LPM model.

Excellent matching has been achieved between the results obtained by the reduced model and the results of the MCS for the FEM model performed in Code_Aster. Non-dimensional formulation of the sensitivity has pointed out the importance of the stiffness of the ViBa in evaluating the stochastic response of the industrial building. Finally, a relevant sensitivity of the response to variation of the rotational stiffness component of LPM has been observed.

\section{Acknowledgments}

The authors Tombari and Cacciola gratefully acknowledge the financial support of EPSRC First Grant EP/K004867/1 “Vibrating Barriers for the control of seismic waves (ViBa)”.

\section{References}

Bampton MCC, Craig, Jr. RR, Coupling of substructures for dynamic analyses, AIAA Journal., 6(7):1313-9 ,Jul;1968.

Benfratello S., Caddemi S., Muscolino G., Gaussian and non-Gaussian stochastic sensitivity analysis of discrete structural system, Computer and Structures 78 425-434, 2000.

Bhattacharyya B., Chakrabborty S., Stochastic dynamic sensitivity of uncertain structures subjected to random earthquake, loading, J. Sound Vibrat. 249, 543-556, 2002. 
Chaudhuri A., Chakraborty S., Sensitivity evaluation in seismic reliability analysis of structures, Comput. Methods Appl. Mech. Engrg. 193, 59-68, 2004.

Cacciola P, Colajanni P, Muscolino G, A modal approach for the evaluation of the response sensitivity of structural systems subjected to non-stationary random processes, Computer Methods in Applied Mechanics and Engineering, 194(42-44):4344-61, Oct 2005.

Cacciola P, EPSRC First Grant EP/K004867/1 “Vibrating Barriers for the control of seismic waves (ViBa)”, 2012.

Cacciola P, Espinosa MG, Tombari A. Vibration control of piled-structures through structure-soilstructure-interaction. Soil Dynamics and Earthquake Engineering. 2015 Oct;77:47-57.

Cacciola, P. and Muscolino, G. Stochastic seismic analysis of large linear structural systems under fully non-stationary spectrum compatible ground motion In: Computational Mechanics in Stochastic Dynamics. Springer, pp. 89-109, 2011

Cacciola, P., Tombari, A., Vibrating barrier: a novel device for the passive control of structures under ground motion. Proceedings of the Royal Society of London A: Mathematical, Physical and Engineering Sciences 471. doi:10.1098/rspa.2015.0075, 2015.

Clouteau D, MISS 6.4. Ecole Centrale de Paris, Chatenay-Malabry, France; 2005.

Crandall, SH, The role of damping in vibration theory, Journal of Sound and Vibration 11, 3-IN1, 1970.

Di Paola, M., Petrucci, G.: Spectral moments and pre-envelope covariances of nonseparable processes. Journal of Applied Mechanics (ASME) 57, 218-224, 1990.

EDF R\&D, Code_Aster [Internet]. EDF R\&D; Available from: http://www.code-aster.org EPRI, Effect of Seismic Wave Incoherence on Foundation and Building Response. EPRI, Report No.: 1013504, 2006.

Fox R.L., Kapoor M.P., Rates of change of eigenvalues and eigenvectors, Am. Inst. Aeronaut. Astronaut. J. 6 2426-2429, 1968. 
Hien T.D., Kleiber M., Stochastic design sensitivity in structural dynamics, Int. J. Numer. Methods Engrg. 32 1247-1265, 1991.

Huang C.D., Socha L., Soong T.T., Stochastic sensitivity analysis of primary-secondary structural systems, Engrg. Struct. 15 (2), 135-142, 1993.

Huang C.D., Soong T.T., Stochastic sensitivity analysis of nonlinear primary-secondary structural systems, Engrg. Struct. 16 (2), 91-96, 1994.

Johnson, E. and Wojtkiewicz, S.”Efficient Sensitivity Analysis of Structures with Local Modifications. II: Transfer Functions and Spectral Densities.” J. Eng. Mech., 140(9), 04014068. (2014).

Kausel E, Whitman R.V, Morray J.P., Elsabee F, The spring method for embedded foundations. Nuclear Engineering and Design, Aug. 1978.

Liu Q., Sensitivity and Hessian matrix analysis of PSD functions for uniformly modulated evolutionary random seismic responses, Finite Elem. Anal. Des., 48, 1370-1375, 2012.

Liu Q., Sensitivity and Hessian matrix analysis of evolutionary PSD functions for nonstationary random seismic responses, J. Eng. Mech. ASCE, 138, 716-720, 2012.

Liu Q. and Paavola J.. Sensitivity and Hessian matrix analysis of structural reliability for uniformly modulated random seismic response, Mechanics Research Communications 62: 155-161, 2014.

Marano, G. C. , Trentadue F, and Morrone E.. Sensitivity analysis of optimum stochastic nonstationary response spectra under uncertain soil parameters. Soil Dynamics and Earthquake Engineering 28.12: 1078-1093, 2008.

Muscolino G., Dynamically modified linear structures: deterministic and stochastic response, J. Engrg. Mech. Div., ASCE 122(11), 1044-1051, 1996.

Neumark S, Concept of complex stiffness applied to problems of oscillation with viscous and hysteretic damping. Aero Research Council R\&M, Report No.: 3269, 1957. 
Sarkar, S and Ghosh D. A hybrid method for stochastic response analysis of a vibrating structure. Archive of Applied Mechanics: 1-20, 2015.

Spanos P.D. and Zeldin B. Pitfalls of Deterministic and Random Analyses of Systems with Hysteresis.” J. Eng. Mech., 126(10), 1108-1110, 2000. 


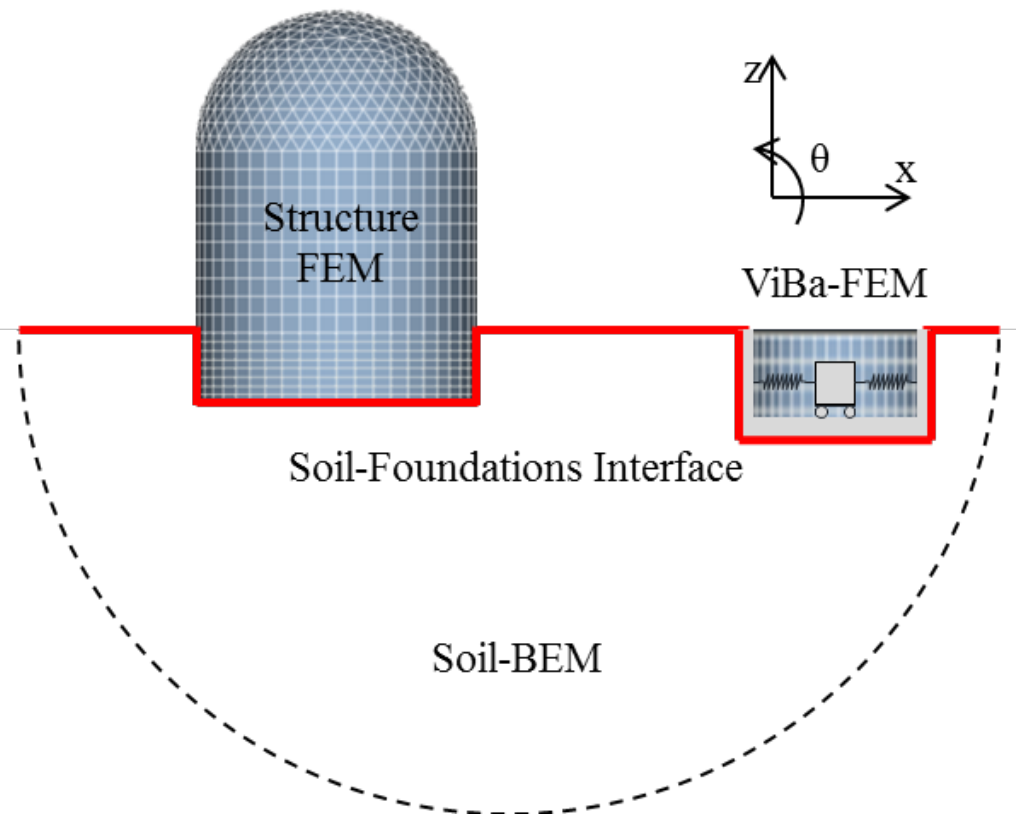

Figure 1 Subdomains of the global problem considered in the paper 
Table 1 Significant dimensions of the industrial building

\begin{tabular}{lc}
\hline industrial building shell radius & $25.8 \mathrm{~m}$ \\
Basement shell radius & $25.8 \mathrm{~m}$ \\
Height of springline above basemat & $46.12 \mathrm{~m}$ \\
Embedded height & $12.9 \mathrm{~m}$ \\
Wall thickness & $1.07 \mathrm{~m}$ \\
Basemat thickness & $3.05 \mathrm{~m}$ \\
\hline
\end{tabular}


Table 2 Significant dimensions of the proposed device ViBa

\begin{tabular}{lc}
\hline Basement shell radius & $12.9 \mathrm{~m}$ \\
Distance from the structure & $12.9 \mathrm{~m}$ \\
Embedded height & $6.45 \mathrm{~m}$ \\
Wall thickness & $1.5 \mathrm{~m}$ \\
Basemat thickness & $1.5 \mathrm{~m}$ \\
\hline
\end{tabular}



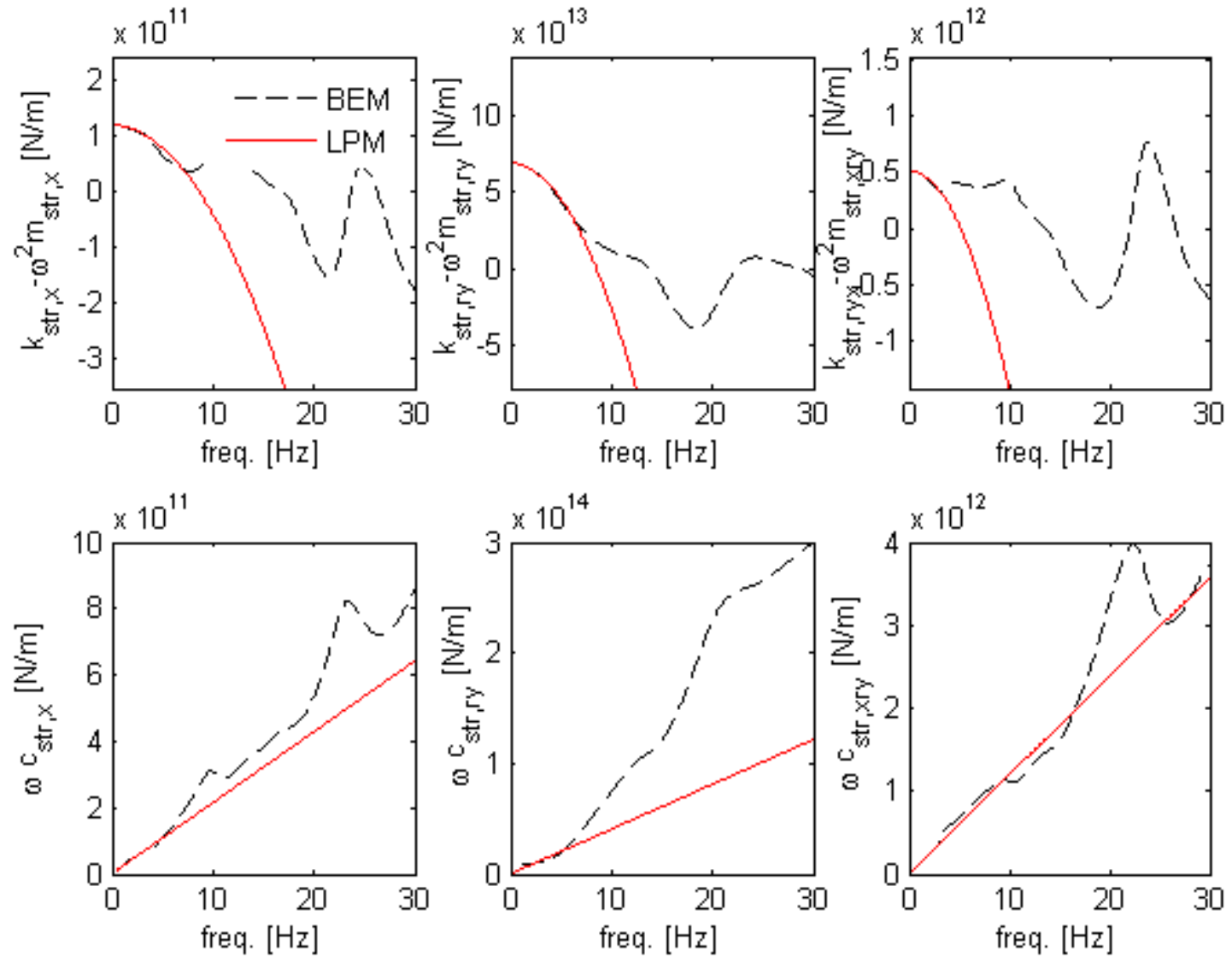

Figure 2 Approximate structural impedances of the LPM compared to those evaluated by BEM 

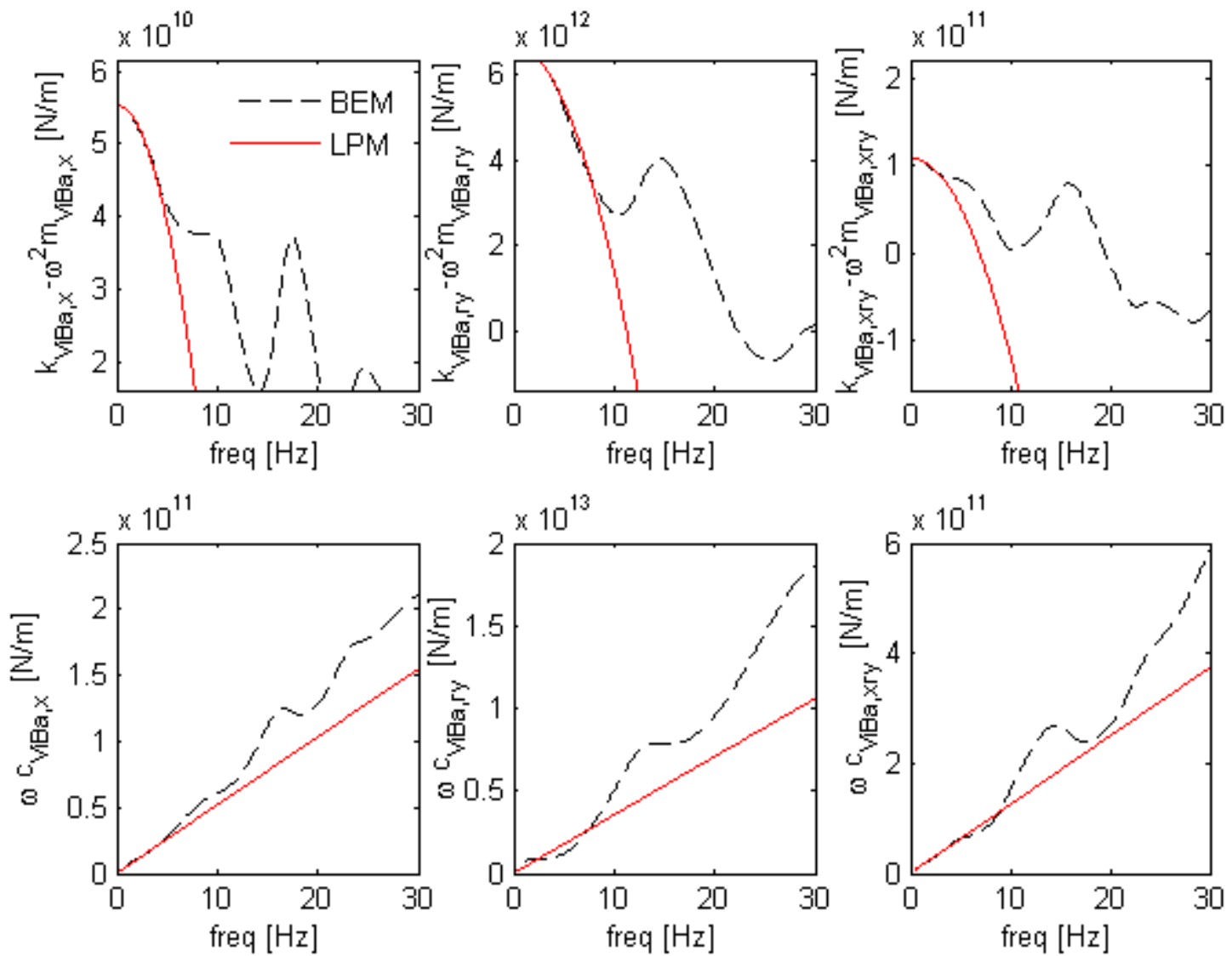

Figure 3 Approximate ViBa impedances of the LPM compared to those evaluated by BEM 

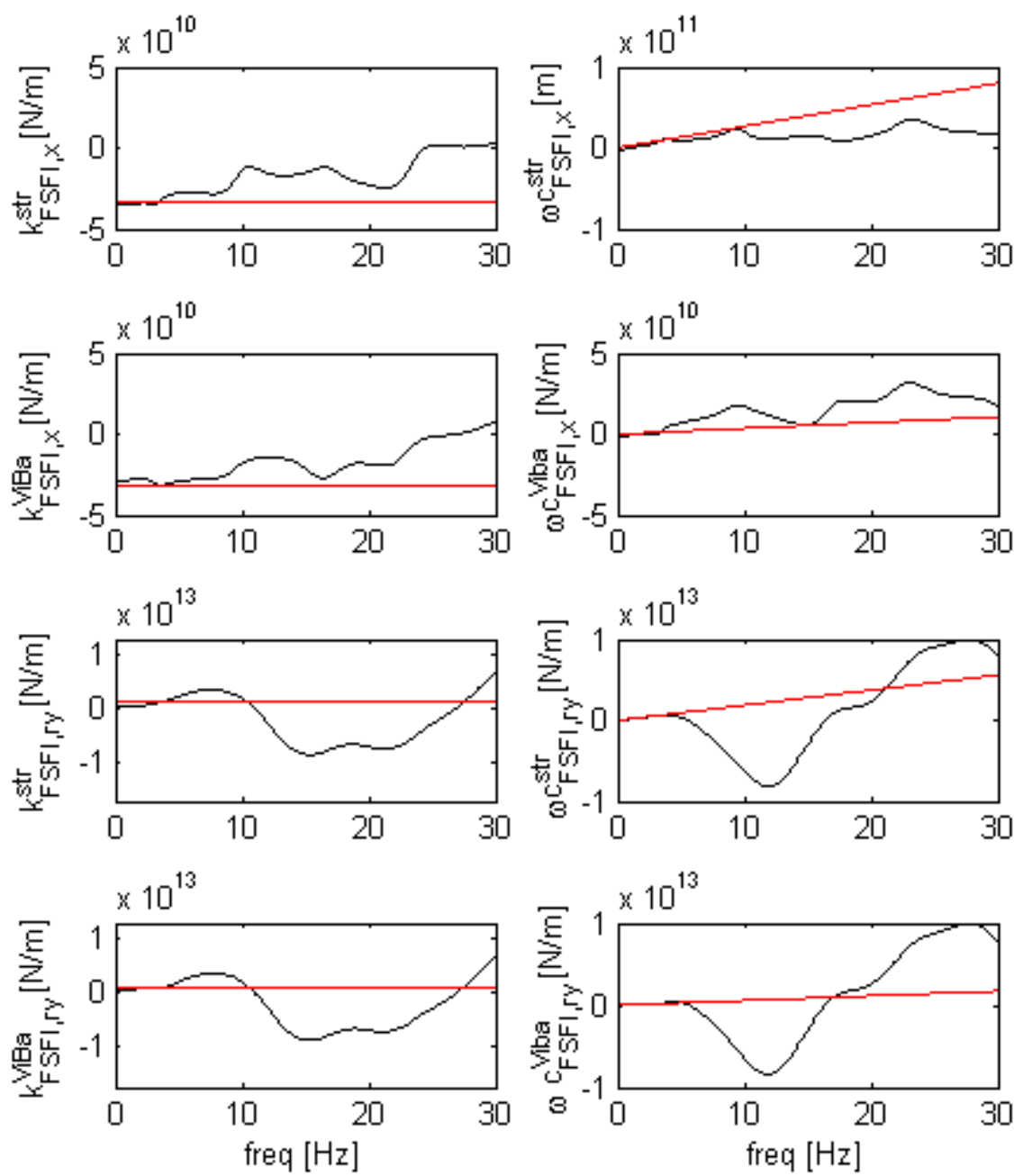

Figure 4 Approximate coupling impedances of the LPM (red curve) compared to those evaluated by BEM (black curve) 

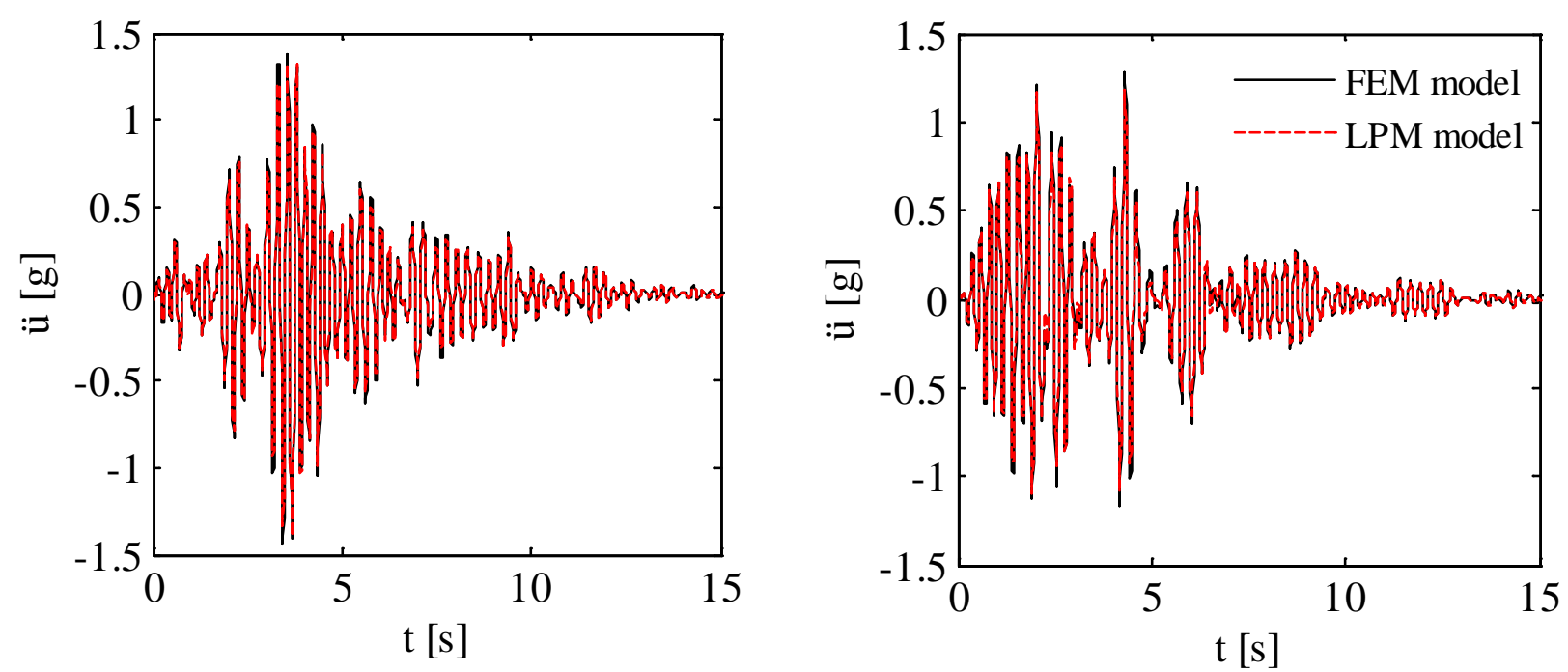

Figure 5 Comparison between two trajectories of the top of the structure obtained from the FEM and the LPM model 


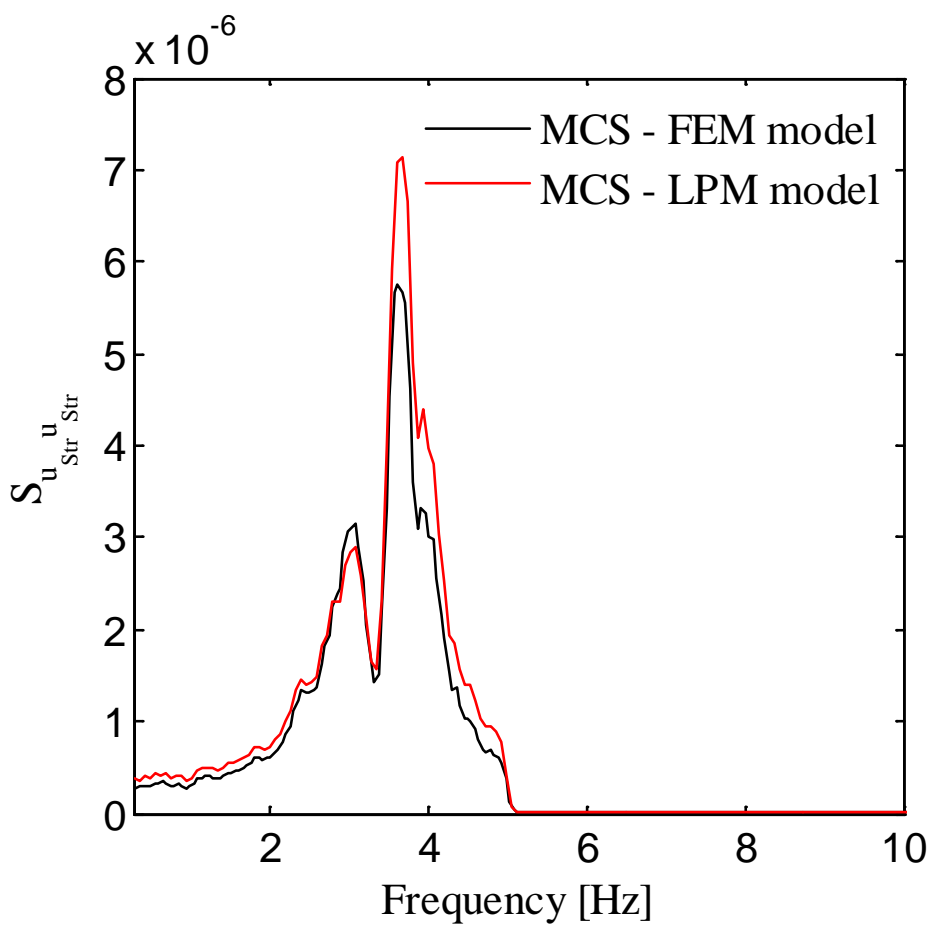

Figure 6 Comparison between power spectral density functions obtained from FEM and LPM models. 

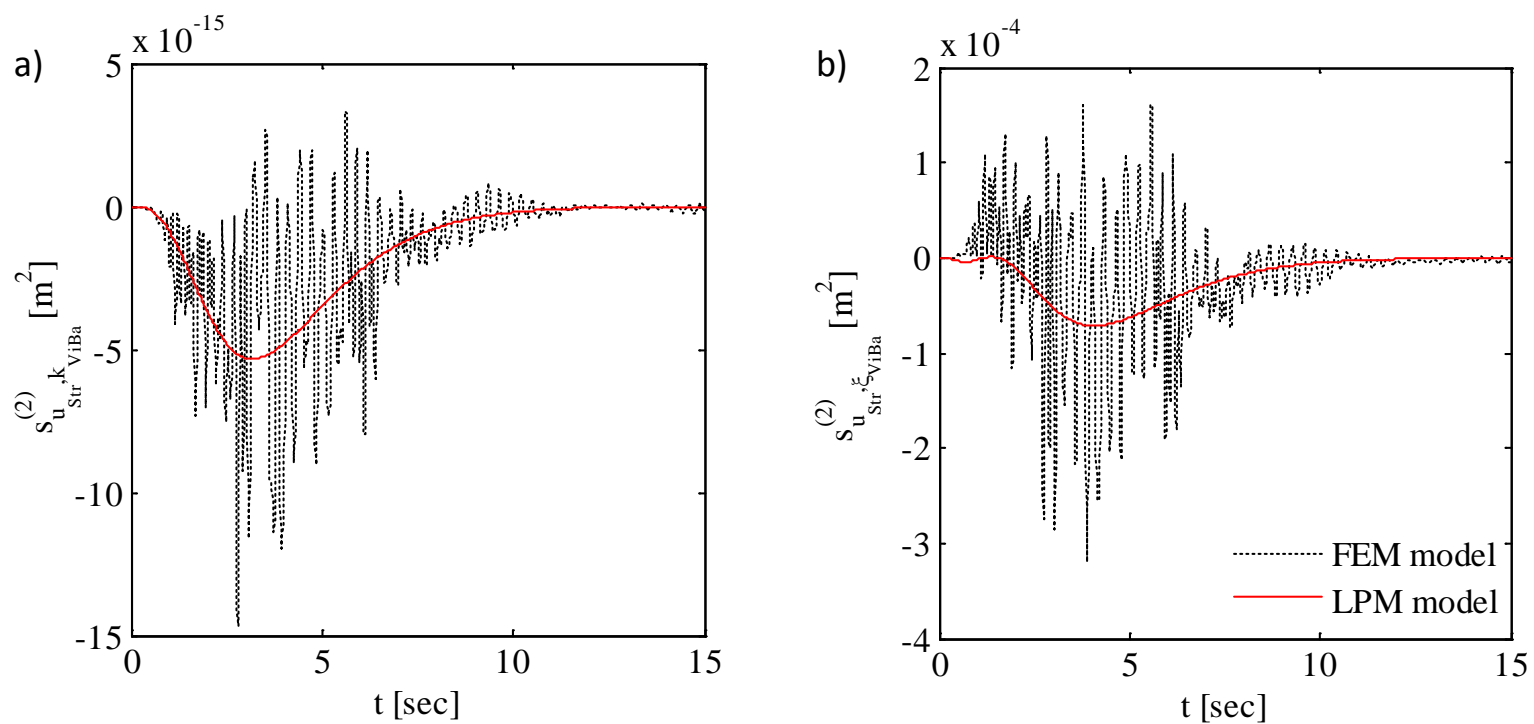

Figure 7 Sensitivity of the second-order statistical moments of the nodal displacement of the structure with respect to the a) ViBa stiffness and b) ViBa damping 

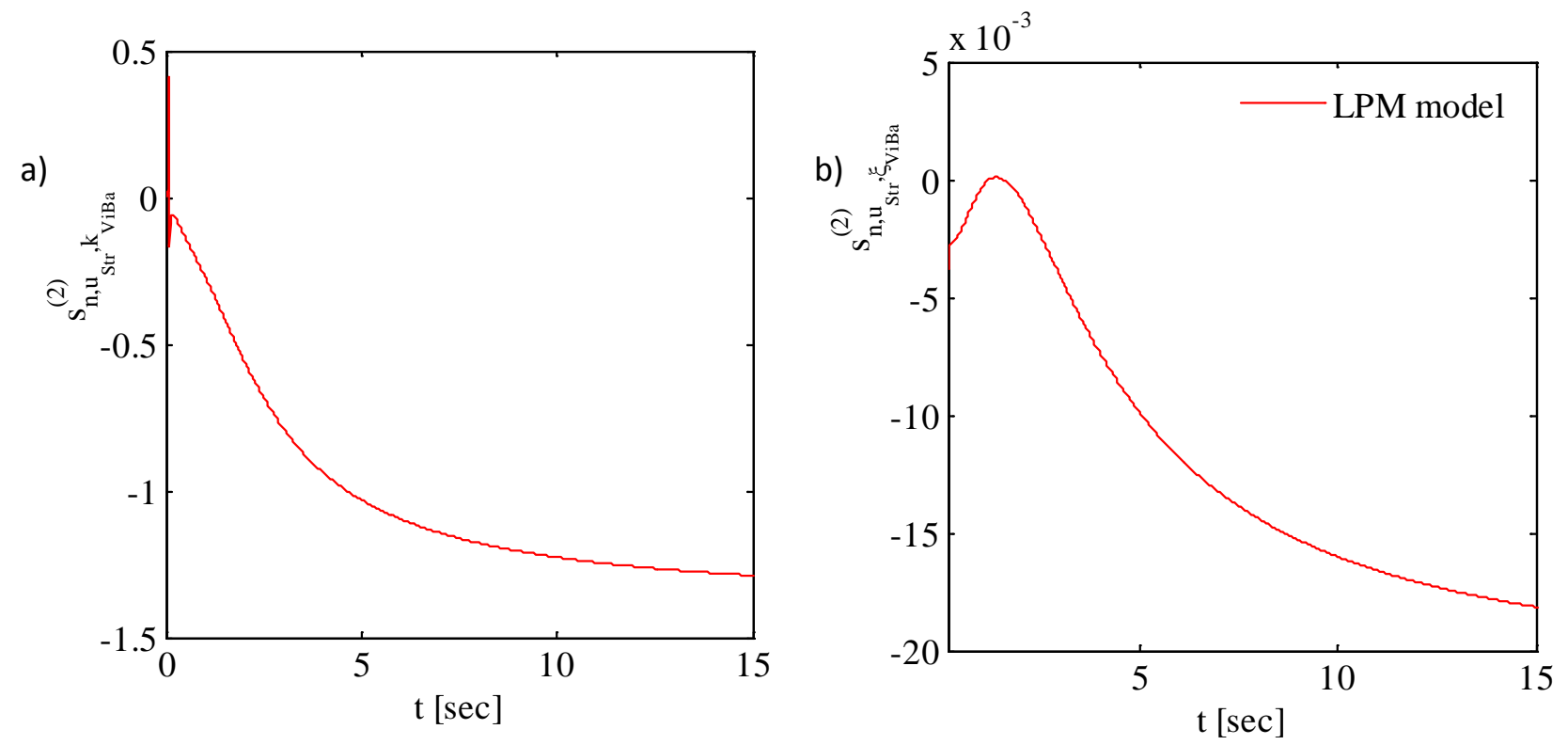

Figure 8 Non-dimensional sensitivity of the second-order statistical moments of the nodal displacement of the structure with respect to the a) stiffness and the b) damping of the ViBa 

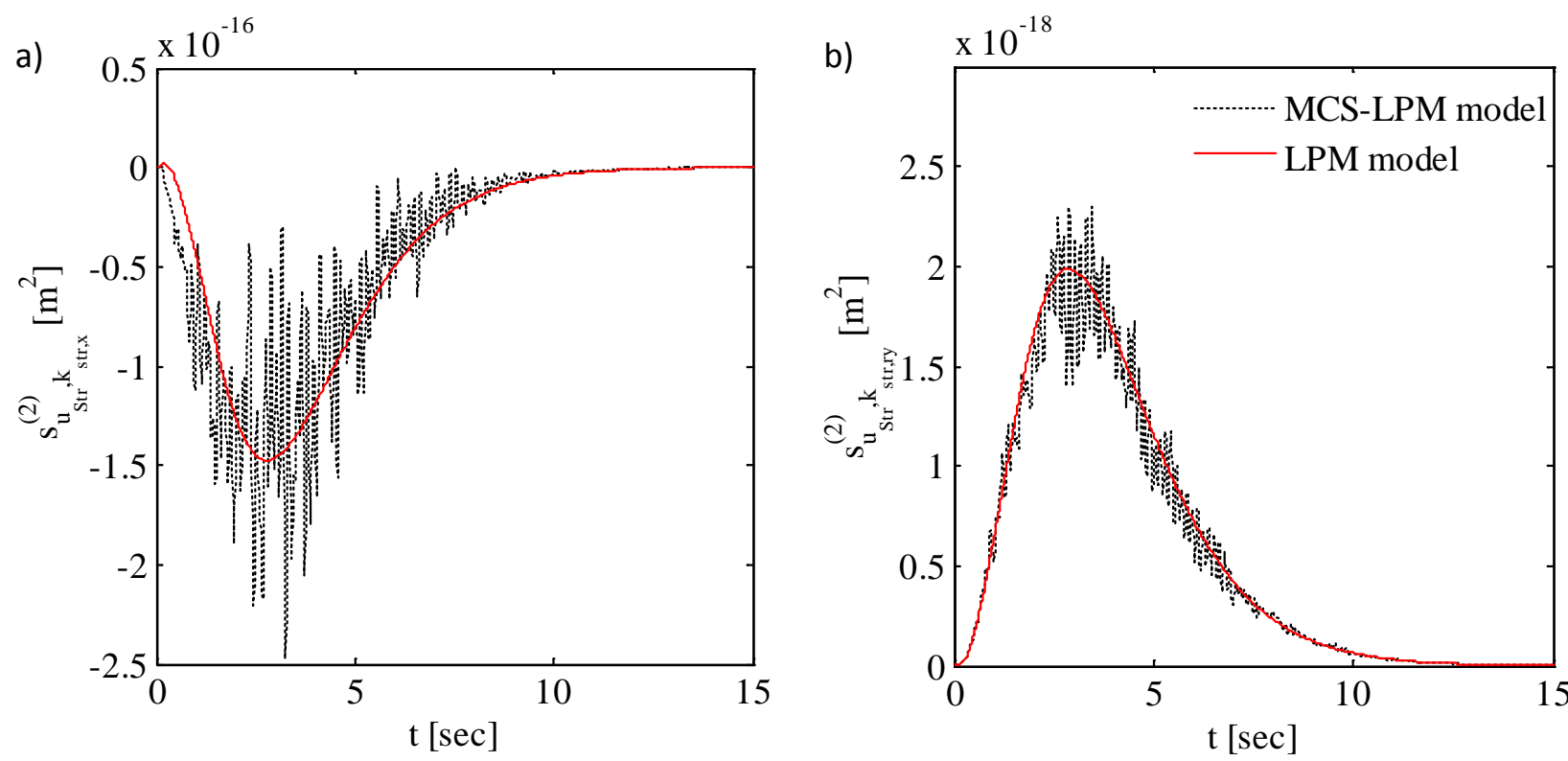

Figure 9 Sensitivity of the second-order moments of the nodal displacement of the structure with respect to the a) $\mathrm{x}$-LPM component and b) ry-LPM component of the stiffness 
a)

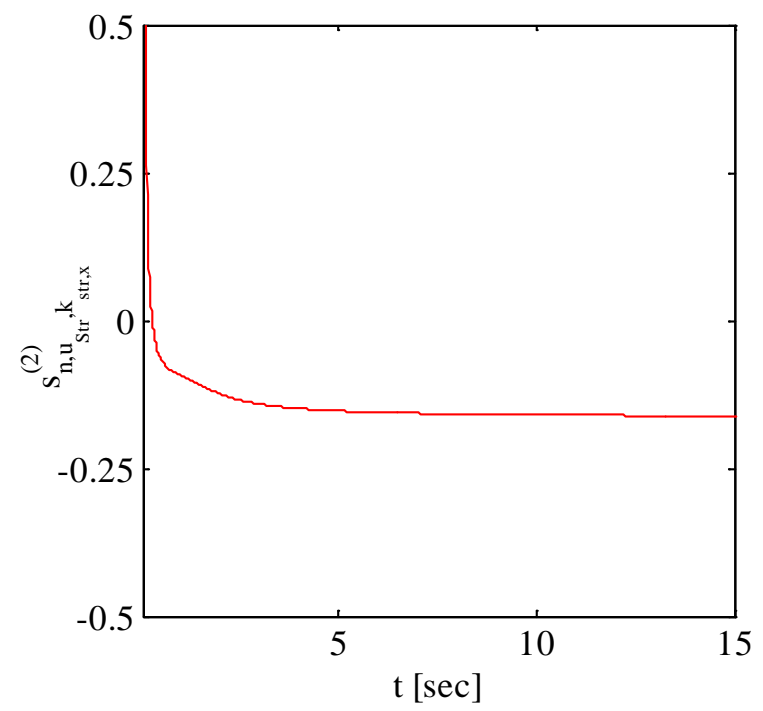

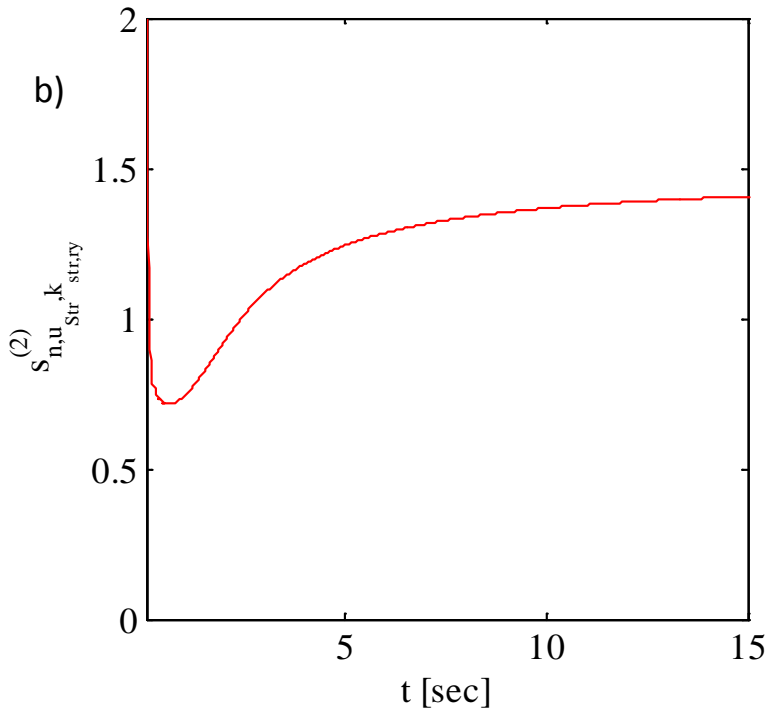

Figure 10 Non-dimensional sensitivity of the second-order statistical moments of the structure with respect to the a) x-LPM component and b) ry-LPM component of the stiffness 